Yerbilimleri, 2020, 41 (1), 1-29, DOI:10.17824/yerbilimleri.684511

Hacettepe Üniversitesi Yerbilimleri Uygulama ve Araştırma Merkezi Bülteni

Bulletin of the Earth Sciences Application and Research Centre of Hacettepe University

\title{
Geochemical differences between bed and terrace sediments of the Harşit Stream (NE Turkey): Implications for mixed source rocks, weathering and mass transfer
}

Harşit Çayına ait yatak ve taraça sedimentleri arasındaki kimyasal farklılıklar (KD Türkiye): Karışık kaynak kayaç, ayrışma ve kütle transferi

\section{ÇIĞDEM SAYDAM EKER ${ }^{1}$}

${ }^{1}$ Department of Geology, Engineering and Natural Sciences Fac., Gümüşhane University, 29000 Gümüşhane, Turkey

Geliş (received) : 4 Şubat (February) 2020

Kabul (accepted) : 17 Nisan (April) 2020

\section{ABSTRACT}

There are minor geochemical differences between Harşit Stream bed and terrace sediments, and these can be owing to the impacts of climatic differences and chemical weathering rather than source rock composition. The bed sediments have lower average $\mathrm{SiO}_{2}, \mathrm{Al}_{2} \mathrm{O}_{3}, \mathrm{Fe}_{2} \mathrm{O}_{3}, \mathrm{~K}_{2} \mathrm{O}, \mathrm{TiO}_{2}$ and $\mathrm{MnO}$ concentrations than terrace sediments, but higher concentrations of $\mathrm{MgO}, \mathrm{CaO}$ and $\mathrm{Na}_{2} \mathrm{O}$. The rare earth element (REE) distributions, $\mathrm{Eu} / \mathrm{Eu}^{*},(\mathrm{Gd} / \mathrm{Yb})_{\mathrm{N}}, \mathrm{La} / \mathrm{Th}$ and $(\mathrm{La} / \mathrm{Yb})_{N}$ ratios of the bed and terrace sediments indicate that they derived from a mixture of $3 \%$ granodiorite $+50 \%$ gabbro $+47 \%$ felsic tuff and $5 \%$ granodiorite $+37 \%$ gabbro $+58 \%$ felsic tuff, respectively. The CIA, PIA, WIP values and $\mathrm{ICV}, \mathrm{Rb} / \mathrm{Sr}$ ratios indicate that the bed sediments are lowly weathered and terrace sediments are lowly to moderately weathered. The distribution of C-values and $\mathrm{Sr} / \mathrm{Cu}$ ratios indicate an arid to semiarid climatic conditions for the bed sediments and an arid to semi-moist climatic conditions for terrace sediments. During weathering of the mixed source rocks, $\mathrm{Na}$ indicates the greatest loss for the bed and the terrace sediments, and $\mathrm{P}$ shows the lowest loss for both of sediments. The average of $\mathrm{Si}, \mathrm{Al}, \mathrm{Na}, \mathrm{K}$ and $\mathrm{Mn}$ exhibits more loss in bed sediments than terrace sediments, the $\mathrm{Fe}, \mathrm{Mg}, \mathrm{Ca}$ and $\mathrm{P}$ exhibits less loss in bed sediments than terrace sediments.

Keywords: NE-Turkey, stream sediment, mixed source, weathering, paleoclimate, mass transfer.

https://doi.org/10.17824/yerbilimleri.684511

$\lambda$ Çiğdem SAYDAM EKER csaydam@gumushane.edu.tr

${ }^{1}$ Gümüşhane Üniversitesi, Mühendislik ve Doğa Bilimleri Fak., Jeoloji Müh. Böl., 29000 Gümüşhane, Türkiye, ORCID 05325863555 


\section{ÖZ}

Harşit Çayı kanal ve taraça sedimentleri arasında küçük jeokimyasal farklııklar vardır. Bu farklılıklar kaynak kaya bileşiminden ziyade kimyasal ayrışma ve iklimsel farklılıklardan dolayı meydana gelmiş olabilir. Kanal sedimentleri, taraçaya göre daha düşük $\mathrm{SiO}_{2}, \mathrm{Al}_{2} \mathrm{O}_{3}$, $\mathrm{Fe}_{2} \mathrm{O}_{3}, \mathrm{~K}_{2} \mathrm{O}, \mathrm{TiO}_{2}$ ve $\mathrm{MnO}$, fakat daha yüksek $\mathrm{MgO}$, CaO ve $\mathrm{Na}_{2} \mathrm{O}$ ortalama konsantrasyonuna sahiptir. Kanal ve taraça sedimentlerinin nadir toprak element dağılımları (NTE), Eu/Eu*, $(G d / Y b)_{N}, L a / T h$ ve $(L a / Y b)_{N}$ oranları, bu sedimentlerin sırasıyla $\% 3$ granodiyorit $+\% 50$ gabro $+\% 47$ felsik tüf ve $\% 5$ granodiyorit $+\% 37$ gabro $+\% 58$ felsik tüften türediğini göstermektedir. CIA, PIA, WIP değerleri ve ICV, Rb/Sr oranları kanal sedimentlerinin düşük seviyede, taraça sedimentlerinin ise düşük - orta seviyede kimyasal ayrışmaya uğradığını göstermektedir. C-değerleri ve Sr/Cu oranlarının dağılımı, kanal sedimentleri için kurak - yarı kurak, taraça sedimentleri için kurak - yarı nemli iklim şartlarının hâkim olduğunu göstermektedir. Karışık kaynak kayaçların ayrışması sırasında, kanal ve taraça sedimentlerinde en çok kayba uğrayan elementin $\mathrm{Na}$, en az kayba uğrayan elementin ise $P$ olduğu tespit edilmiştir. Kanal sedimentlerinin ortalama Si, Al, Na, $K$ ve $\mathrm{Mn}$ elementleri taraçaya göre daha fazla, Fe, $\mathrm{Mg}$, Ca ve $P$ elementleri ise daha az kayba uğramıştır.

Anahtar kelimeler: KE-Türkiye, dere sedimanı, karışık kaynak, ayrışma, paleoiklim, kütle transferi.

\section{INTRODUCTION}

The siliciclastic sedimentary rocks and detrital sediments preserve detailed evidence of the Paleozoic, Mesozoic and Cenozoic tectonic and climatic history of the Gümüşhane are and related region. Chemical composition of siliciclastic sedimentary rocks and detritic sediments is controlled by several factors from source rocks composition, erosion, chemical and physical weathering, transport, deposition and diagenesis (Johnsson, 1993; Jian et al., 2013). Because, weathering by chemical and physical alteration of available rocks and minerals forms detrital sediments. Former studies have indicated that the major elements of sediments can be used as determiners of source rock property, tectonic setting, provenance of the sediments changes in the supply of material from different sources (Bhatia, 1983; Taylor and McLennan, 1985; Rosher and Korsch, 1986; 1987; Wronkiewicz and Condie, 1987; McLennan, 1989; McLennan and Taylor, 1991; McLennan, et al., 1993; Roddaz et al., 2011; Armstrong-Altrin, et al., 2017; 2018). Nevertheless, major elements abundances are altered by chemical weathering in the source area (Nesbitt, 1979; McLennan, 1989), and also their compositional variations that 
happen during chemical weathering can be performed to evaluate the weathering history of sediments and paleoclimatic conditions (Nesbitt et al., 1980; Nesbitt and Young, 1982; McLennan, et al., 1993; Fedo et al., 1996; 1997; Young, 1999; Jian et al., 2013). The proportion of mafic and felsic contributions to sediments can be differentiated using provenance sensible indicators, like ratios of immobile elements ( $\mathrm{La}, \mathrm{Sc}, \mathrm{Co}, \mathrm{Th})$ and Rare Earth Elements (REEs) (Zhou et al., 2017).

Rivers are the main sources of detrital particles delivered to various basins and enforce a major control on the formation of sedimentary rocks. The proficiency of rivers to transport sediment is directed by their flow, gradient, volume and the sediment load itself. Characteristics of a river basin like geology, geomorphology, climate and vegetation are significant factors controlling the composition and amounts of materials carried by rivers (Milliman and Meade, 1983; Kaotekwar et al., 2019). Mineralogical and chemical compositions of river deposits are highly useful in understanding the geochemical cycling of elements and erosional properties of river basins (Martin and Meybeck, 1979; Milliman and Meade, 1983; Kaotekwar et al., 2019). Lithology, diagenesis, weathering and sedimentary sorting of catchment areas control major, trace and rare earth element (REE) compositions of river sediments and so can be used to identify provenance of sediments (Piper, 1974; Alexander and Gibbson, 1977; Condie, 1991; Sensarma et al., 2008; Kessarkar et al., 2010). Terrace sediments overlain on the base of a sequences boundary are widespread in fluvial sediments of a river or stream valley. Nevertheless, terrace sediments are usually ignored because their volume is small to negligible compared with other formations. However, terrace sediments often contain important geological information. Terrace sediments reveal differences of climatic conditions, chemical weathering and source rocks between present day and a few hundreds years ago. The purpose of this study is to compare mixed of source rock compositions, weathering intensity and quantifications of mass transfer values of the Harşit Stream (Gümüşhane, NE Turkey) bed and terrace sediments. The thickness of the terrace sediments ranges from 2 to 6 meters and it has a well-developed terrace system (Saydam Eker, 2017)

\section{GEOLOGICAL SETTING AND CLIMATE}

The studied sediments are located in the region Gümüşhane, in the Eastern Pontides, NE Turkey. The Eastern Pontides orogenic belt is commonly subdivided into southern and northern parts that change in terms of rock groups. The southern part is a multi-phase tectonic unit, containing plutonic, volcanic, metamorphic and sedimentary rocks, and the northern part is dominated by Carboniferous to Eocene granitoids and volcanic rocks (Eyuboğlu, 2015). The basement of the north-eastern Pontides contains Lower Carboniferous metamorphic rocks (Topuz et al., 2004) and granitoids of Upper Carboniferous age (Çoğulu, 1975; Topuz et al., 2010; Dokuz, 2011; Kaygusuz et al., 2012; 
Kaygusuz et al., 2016). The Early and Middle Jurassic volcaniclastic, sedimentary and volcanic rocks (conglomerates, sandstones, carbonates, gabbros, granites, basalts and rhyolites) lie unconformably on this basement. The Jurassic rocks are conformably overlain by neritic and pelagic carbonates of Upper Jurassic to Middle Cretaceous units. Late Cretaceous rocks are represented by turbidites in the south-eastern Pontides (Saydam Eker and Korkmaz, 2011). Paleocene and Eocene rocks in the Gümüşhane region comprise granite and volcanic (Kaygusuz et al., 2011; Aslan et al., 2014), volcanosedimentary and sedimentary rocks. This unit is wide in the region and discordantly overlies the elder rocks (Fig. 1).

The basement rocks of the stream basin are composed of Carboniferous plutons. Lower Middle Jurassic volcanites and volcaniclastic, clastic deposits lie unconformably on the Carboniferous plutons. This unit is overlain by platform carbonates of the Early JurassicLower Cretaceous age. The Upper Cretaceous sedimentary rocks lie conformably on the platform carbonates. Eocene rocks in this basin are composed of andesites and dacites and lie unconformably on the Upper Cretaceous unit (Fig. 2A).
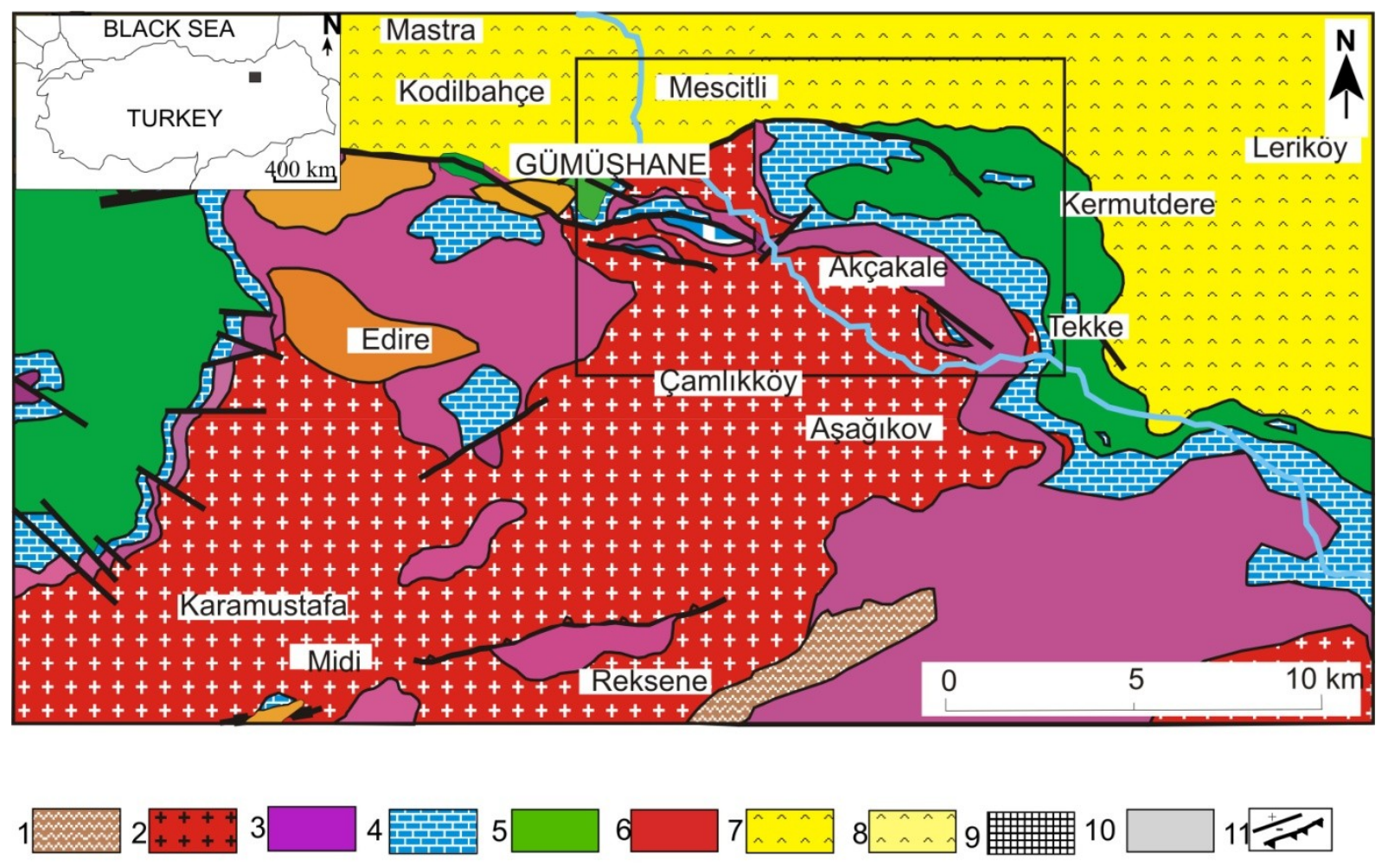

Figure 1. Geological map of Gümüşhane and its vicinity (modified from Güven et al., 1993) and location map of the study area. 1- Paleozoic metamorphic rocks, 2- Paleozoic granitoid, 3- Jurassic volcanic, volcanoclastic and sedimentary rocks, 4- Lower Cretaceous platform carbonates, 5- Upper Cretaceous volcanic and sedimentary rocks, 6- Paleocene granites, 7- Eocene andesites, 8- Eocene dacites, 9Travertines, 10. Alluviums, 11- Thrust fault and fault.

Şekil 1. A. Gümüşhane ve çevresinin jeolojik haritası (Güven vd., 1993'den değiştirilerek) ve çalışma alanının lokasyon haritası. 1- Paleozoyik metamorfik kayaçları, 2- Paleozoyik granotoidi, 3- Jura volkanik, volkanoklastik ve sedimanter kayaçları, 4- Alt Kretase platform karbonatları, 5- Üst Kretase volkanik ve sedimanter kayaçları, 6- Paleosen granitleri, 7- Eosen andezitleri, 8- Eosen dasitleri, 9- Travertenler, 10Alüvyonlar, 11- Bindirme fayı ve fay. 
Climate at the studied area is essentially moderate. The summer months (June to August) are dominated by the heat (The average temperature is approximately $30^{\circ} \mathrm{C}$ ). Rainy season is seen between March and June, and between September and November. The average annual rainfall in the studied location covers approximately $400 \mathrm{~mm}$. The humidity rises up to $70-88 \%$ during rainy season. The average temperature is approximately $-1{ }^{\circ} \mathrm{C}$ in winter months (December to February) (Climate-Data-Org., 2020).

\section{MATERIAL AND METHODS}

\section{Sampling and preparation}

A total of 49 samples were collected throughout the Harşit Stream (between the Mescitli and Tekke areas), 35 samples for bed sediments and 14 samples for terrace sediments. The sampling locations of collected bed and terrace sediments are displayed in Fig. 2B. The sediment samples were collected in September as representatives of low-flow conditions. The samples of the bed sediments were achieved from the stream bed. The vertical face of the terrace was trenched inward by approximately $45 \mathrm{~cm}$ to $50 \mathrm{~cm}$ and the 14 samples were obtained from there. The samples were air dried in the laboratory prior to analytical analysis and then homogenized split into sub-samples and crushed to roughly $0.075 \mathrm{~mm}$ (200 meshes) size using an agate mill (Saydam Eker, 2017).

\section{Analytical techniques}

Major, trace, and REE elements were determined at ACME Analytical Laboratories Ltd., Vancouver, Canada. Major and trace element compositions were measured by inductively coupled plasma (ICP)-emission spectrometry and ICP-mass spectrometry (MS) using 0.2 $\mathrm{g}$ of rock powder fused with $1.5 \mathrm{~g}$ of $\mathrm{LiBO}_{2}$ dissolved in $100 \mathrm{ml}$ of $5 \% \mathrm{HNO}_{3}$. Ignition loss was determined on dried samples heated to a temperature of $1000{ }^{\circ} \mathrm{C}$ for $15 \mathrm{~min}$. REE analysis was conducted by ICP-MS at ACME. The detection limits are in the range of 0.001 to $0.1 \mathrm{wt}$ \% for major element oxides, 0.1 to $10 \mathrm{ppm}$ for trace elements, and 0.01 to $0.5 \mathrm{ppm}$ for REE. Calibration and verification standards together with reagent blanks were added to sample sequences. STD SO 18 was certified in-house against 38 certified reference materials including CANMET SY-4 and USGS AGV-1, G-2, GSP-2 and W-2 as external standards. The analytical accuracy is better than $4 \%$. 


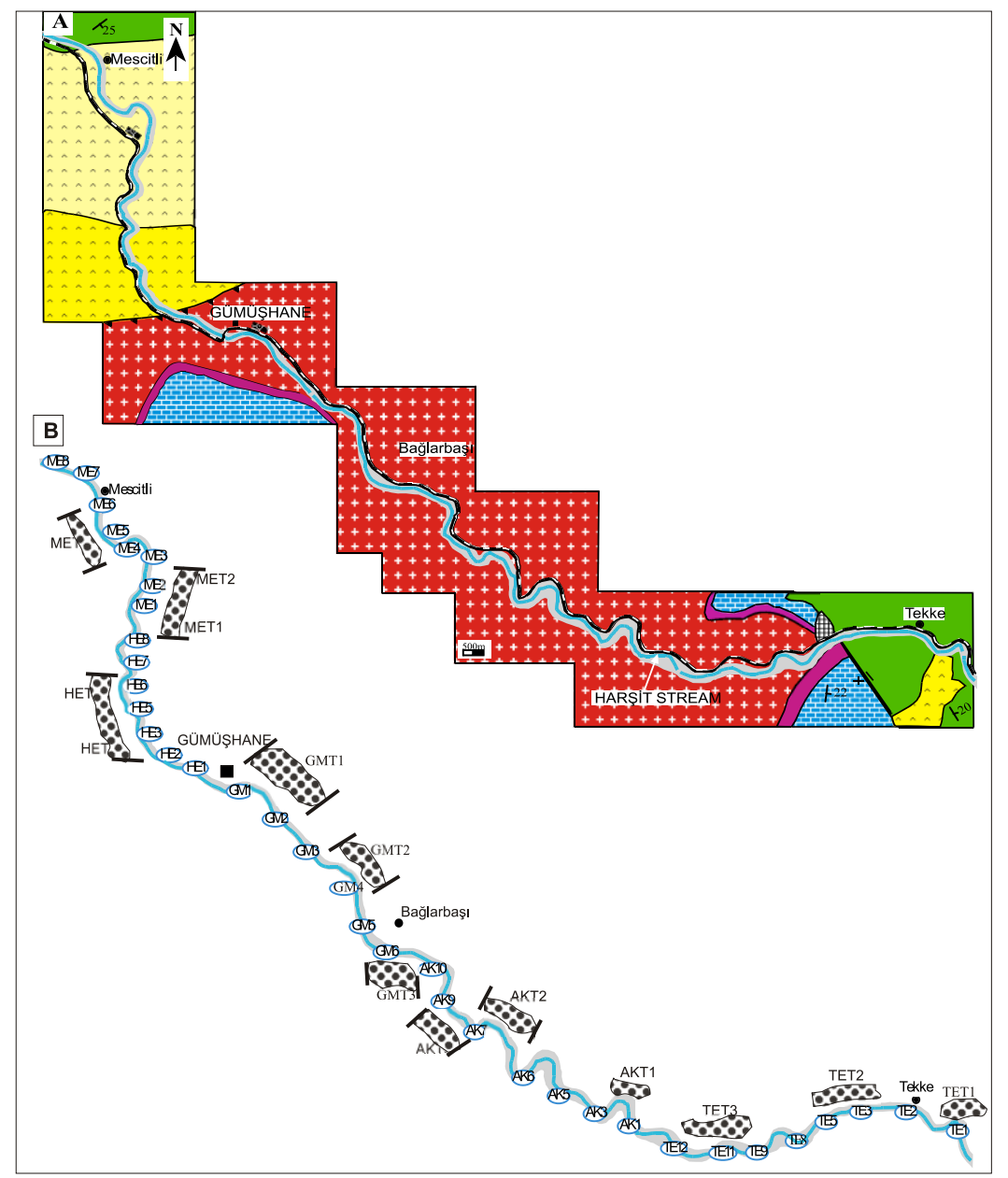

Figure 2. A) Geological map of the study area (Saydam Eker, 2017), B) Sampling sites of stream bed and terrace sediments.

Şekil 2. A) Çalışma alanının jeolojik haritası (Saydam Eker, 2017), B) Kanal ve taraça sedimanları örnekleme yerleri.

In this study, whole rock major element, trace element, and rare earth element (REE) analyses were done on 35 bed sediment samples and 14 terrace sediment samples (Saydam Eker, 2017).

\section{Statistical methods}

Distribution of the data is non-normal, so Spearman's correlation coefficient analysis was performed with statistical software SPSS 22.0 for Windows. Data preparation was calculated through Microsoft Office Excel 2013 and a two-tail test $(p<0.05$ and 0.01$)$ was performed to analyze the variance.

\section{Mixed source composition model}

The objective of the modeling is to reach a mass balance among the REEs and Th/Sc ratio, because, REEs and Th/Sc ratio are sensitive to bulk source composition (Taylor and McLennan, 1985; McLennan and Taylor, 1991; Fedo et al., 1997; Schoenborn and Fedo, 2011; Jian et al., 2013). Major, selected trace and rare earth element data of variety 
potential sources were gathered and used to model the relative proportions of end members that subscribed sediment to the Harşit Stream in the Gümüşhane region. Endmembers used in the mixing computation were selected from the distinctive igneous and volcanic rocks in the Gümüşhane area. The end member is a $320 \mathrm{Ma}$ granodiorite exposed in the Gümüşhane region (Topuz et al., 2010), the second end member is a 177 Ma gabbro (Karslı et al., 2017) and the third end-member is a 84 Ma felsic tuffs (Eyuboğlu, 2015).

\section{RESULTS}

\section{Geochemical characteristics}

The major, selected trace and rare earth element composition of the Harşit bed and terrace sediments are listed in Table 1 . The bed sediment samples have lower average $\mathrm{SiO}_{2}, \mathrm{Al}_{2} \mathrm{O}_{3}, \mathrm{Fe}_{2} \mathrm{O}_{3}, \mathrm{~K}_{2} \mathrm{O}, \mathrm{TiO}_{2}$ and $\mathrm{MnO}$ concentrations than samples from terrace sediment, but higher concentrations of $\mathrm{MgO}, \mathrm{CaO}$ and $\mathrm{Na}_{2} \mathrm{O}$ (Table 1). When the major, trace and rare earth element components of the bed and terrace sediments are compared upper continental crust (Taylor and McLennan, 1985), the bed sediments are generally enriched in $\mathrm{Fe}_{2} \mathrm{O}_{3}, \mathrm{MgO}, \mathrm{CaO}, \mathrm{Cu}, \mathrm{Sc}, \mathrm{V}, \mathrm{Gd}, \mathrm{Tb}, \mathrm{Ho}, \mathrm{Yb}$ and $\mathrm{Lu}$, and depleted in $\mathrm{Na}_{2} \mathrm{O}$, $\mathrm{K}_{2} \mathrm{O}, \mathrm{MnO}, \mathrm{Rb}, \mathrm{Ba}, \mathrm{Th}, \mathrm{Zr}, \mathrm{Hf}, \mathrm{Y}, \mathrm{Nb}$, La, Ce, Pr, Nd, Sm, Eu, Dy, Er and Tm, and almost similar in $\mathrm{SiO}_{2}, \mathrm{Al}_{2} \mathrm{O}_{3}, \mathrm{TiO}_{2}, \mathrm{P}_{2} \mathrm{O}_{5}, \mathrm{Sr}, \mathrm{U}$, Co. The terrace sediments are usually enriched in $\mathrm{F}_{2} \mathrm{O}_{3}, \mathrm{MgO}, \mathrm{TiO}_{2}, \mathrm{Cu}, \mathrm{Sc}, \mathrm{V}, \mathrm{Yb}$ and $\mathrm{Lu}$, and depleted in $\mathrm{Na}_{2} \mathrm{O}, \mathrm{K}_{2} \mathrm{O}, \mathrm{P}_{2} \mathrm{O}_{5}, \mathrm{MnO}, \mathrm{Rb}, \mathrm{Ba}$, $\mathrm{Th}, \mathrm{Zr}, \mathrm{Hf}, \mathrm{Nb}, \mathrm{Ni}$, La, Ce, $\mathrm{Pr}, \mathrm{Nd}, \mathrm{Sm}$, Dy, Er and Tm, and nearly similar in $\mathrm{SiO}_{2}, \mathrm{Al}_{2} \mathrm{O}_{3}$, $\mathrm{CaO}, \mathrm{U}, \mathrm{Y}, \mathrm{Co}, \mathrm{Eu}, \mathrm{Gd}$ and Tb (Saydam Eker, 2017).

For the Harşit bed sediments, $\mathrm{MgO}$ is positively correlated with $\mathrm{CaO}, \mathrm{P}_{2} \mathrm{O}_{5}$ and TOT/C, but negatively correlated to $\mathrm{SiO}_{2}, \mathrm{Na}_{2} \mathrm{O}$ and $\mathrm{K}_{2} \mathrm{O}$, suggesting the occurrence of $\mathrm{Mg}$ in carbonate and apatite. Likewise, $\mathrm{CaO}$ is positively correlated to TOT/C and $\mathrm{P}_{2} \mathrm{O}_{5}$, but negatively correlated to $\mathrm{SiO}_{2}, \mathrm{Na}_{2} \mathrm{O}$ and $\mathrm{K}_{2} \mathrm{O}$. $\mathrm{Fe}_{2} \mathrm{O}_{3}$ exhibits a positive correlation with $\mathrm{TiO}_{2}, \mathrm{MnO}$ and $\mathrm{Al}_{2} \mathrm{O}_{3}$, but negatively correlated to TOT/C, indicating a primary contribution of mafic minerals. $\mathrm{Na}_{2} \mathrm{O}$ and $\mathrm{K}_{2} \mathrm{O}$ are positively correlated to $\mathrm{SiO}_{2}$, but negatively correlated to TOT/C and $\mathrm{P}_{2} \mathrm{O}_{5}$, suggesting the occurrences of $\mathrm{Na}$ and $\mathrm{K}$ in silicates. In addition to, CIA and PIA demonstrate a positive correlation with $\mathrm{SiO}_{2}, \mathrm{Al}_{2} \mathrm{O}_{3}, \mathrm{Na}_{2} \mathrm{O}$ and $\mathrm{K}_{2} \mathrm{O}$, but a negative correlation with $\mathrm{MgO}, \mathrm{CaO}$ and TOT/C. For the terrace sediments, $\mathrm{MgO}$ exhibits a positive correlation with $\mathrm{CaO}$, but a negative correlation with $\mathrm{SiO}_{2}$ and $\mathrm{K}_{2} \mathrm{O}$, suggesting the occurrence of $\mathrm{Mg}$ in carbonate. $\mathrm{CaO}$ is positively correlated to TOT/C and $\mathrm{P}_{2} \mathrm{O}_{5}$, negatively correlated to $\mathrm{SiO}_{2}$. $\mathrm{Fe}_{2} \mathrm{O}_{3}$ is positively correlated to $\mathrm{TiO}_{2}, \mathrm{MnO}$ and $\mathrm{Al}_{2} \mathrm{O}_{3}$ but negatively correlated to $\mathrm{K}_{2} \mathrm{O}$, indicating a primary contribution of mafic minerals. $\mathrm{Na}_{2} \mathrm{O}$ exhibits a positive correlation with $\mathrm{SiO}_{2}$ and $\mathrm{TiO}_{2}$, but a negative correlation with $\mathrm{K}_{2} \mathrm{O}$ and TOT/C, suggesting the occurrences of $\mathrm{Na}$ in silicates and mafic minerals. $\mathrm{K}_{2} \mathrm{O}$ exhibits a negative correlation with $\mathrm{Fe}_{2} \mathrm{O}_{3}, \mathrm{MgO}, \mathrm{Na}_{2} \mathrm{O}, \mathrm{TiO}_{2}, \mathrm{P}_{2} \mathrm{O}_{5}$ and $\mathrm{MnO}$, but there are no correlation with $\mathrm{SiO}_{2}, \mathrm{Al}_{2} \mathrm{O}_{3}$ and TOT/C suggesting the occurrence of K-feldspar. $\mathrm{CIA}$ and $\mathrm{PIA}$ are positively correlated to $\mathrm{K}_{2} \mathrm{O}$, but negatively correlated to $\mathrm{MgO}, \mathrm{CaO}$ and $\mathrm{TiO}_{2}$ (Table 2). 
Table 1 Chemical composition (major, some trace and REE from Saydam Eker, 2017) of analyzed samples. Çizelge 1. Analiz edilen örneklerin kimyasal bileşenleri (ana, bazı iz ve NTE, Saydam Eker, 2017).

\begin{tabular}{|c|c|c|c|c|c|c|c|c|c|c|c|c|}
\hline Sample & $\mathrm{SiO}_{2}$ & $\mathrm{Al}_{2} \mathrm{O}_{3}$ & $\mathrm{Fe}_{2} \mathrm{O}_{3}$ & $\mathrm{MgO}$ & $\mathrm{CaO}$ & $\begin{array}{c}\mathrm{Na}_{2} \mathrm{O} \\
\% \\
\end{array}$ & $\mathrm{~K}_{2} \mathrm{O}$ & $\mathrm{TiO}_{2}$ & $\mathrm{P}_{2} \mathrm{O}_{5}$ & $\mathrm{MnO}$ & $\mathrm{Cr}_{2} \mathrm{O}_{3}$ & TOT/C \\
\hline TE1 Bed & 59.23 & 15.45 & 7.42 & 3.27 & 9.12 & 2.33 & 2.07 & 0.79 & 0.19 & 0.12 & 0.004 & 1.81 \\
\hline TE3 & 61.85 & 15.37 & 6.67 & 2.89 & 7.48 & 2.49 & 2.29 & 0.71 & 0.14 & 0.11 & 0.004 & 1.29 \\
\hline TE4 & 58.68 & 15.6 & 6.95 & 3.47 & 9.75 & 2.56 & 1.97 & 0.73 & 0.16 & 0.12 & 0.005 & 1.68 \\
\hline TE8 & 59.7 & 16.88 & 6.98 & 3.1 & 8.06 & 2.43 & 1.91 & 0.69 & 0.14 & 0.11 & 0.004 & 1.24 \\
\hline TE9 & 62.94 & 15.52 & 6.12 & 2.6 & 6.85 & 2.69 & 2.4 & 0.64 & 0.13 & 0.11 & 0.003 & 1.15 \\
\hline TE11 & 58.95 & 16.27 & 7.12 & 3.36 & 9.13 & 2.33 & 1.83 & 0.75 & 0.16 & 0.11 & 0.005 & 1.58 \\
\hline TE12 & 60.95 & 14.92 & 7.47 & 2.92 & 7.98 & 2.42 & 2.28 & 0.79 & 0.14 & 0.12 & 0.005 & 1.39 \\
\hline AK1 & 62 & 14.74 & 6.16 & 2.69 & 8.58 & 2.48 & 2.48 & 0.63 & 0.13 & 0.1 & 0.006 & 1.88 \\
\hline AK3 & 60.74 & 14.32 & 5.67 & 3.35 & 10.4 & 2.24 & 2.41 & 0.62 & 0.12 & 0.1 & 0.007 & 2.23 \\
\hline AK5 & 64.55 & 14.53 & 6.86 & 2.33 & 5.7 & 2.73 & 2.41 & 0.67 & 0.12 & 0.11 & 0.005 & 0.89 \\
\hline AK6 & 64.42 & 14.66 & 5.09 & 2.54 & 7.51 & 2.65 & 2.4 & 0.51 & 0.12 & 0.09 & 0.004 & 1.32 \\
\hline AK7 & 58.43 & 15.27 & 6.63 & 3.31 & 11.3 & 2.19 & 1.94 & 0.7 & 0.15 & 0.11 & 0.006 & 2.18 \\
\hline AK9 & 62.89 & 14.59 & 6.16 & 2.74 & 7.87 & 2.58 & 2.28 & 0.64 & 0.12 & 0.11 & 0.004 & 1.40 \\
\hline AK10 & 60.1 & 14.87 & 5.98 & 3 & 10.8 & 2.26 & 2.08 & 0.61 & 0.13 & 0.1 & 0.005 & 2.03 \\
\hline GM6 & 63.63 & 15.38 & 5.8 & 2.57 & 6.52 & 2.68 & 2.62 & 0.59 & 0.12 & 0.1 & 0.005 & 1.51 \\
\hline GM5 & 63.86 & 15.88 & 6.39 & 1.97 & 4.81 & 3.07 & 3.16 & 0.64 & 0.12 & 0.1 & 0.004 & 1.16 \\
\hline GM4 & 66.25 & 14.35 & 6.41 & 1.99 & 4.64 & 2.83 & 2.7 & 0.62 & 0.11 & 0.11 & 0.005 & 0.69 \\
\hline GM3 & 58.17 & 16.09 & 6.43 & 3.46 & 10.1 & 2.18 & 2.53 & 0.72 & 0.16 & 0.13 & 0.007 & 2.91 \\
\hline GM2 & 60.18 & 15.51 & 6.14 & 3.37 & 9.04 & 2.34 & 2.49 & 0.67 & 0.15 & 0.1 & 0.007 & 1.96 \\
\hline GM1 & 61.67 & 15.11 & 6.19 & 3.02 & 8.03 & 2.33 & 2.75 & 0.65 & 0.13 & 0.11 & 0.007 & 2.08 \\
\hline HE1 & 58.29 & 15.23 & 5.94 & 3.4 & 11.5 & 2.27 & 2.47 & 0.65 & 0.17 & 0.11 & 0.007 & 2.63 \\
\hline HE2 & 59.9 & 15.01 & 5.83 & 3.28 & 10.3 & 2.32 & 2.47 & 0.63 & 0.16 & 0.1 & 0.007 & 2.31 \\
\hline HE3 & 63.61 & 14.5 & 5.87 & 2.73 & 7.34 & 2.5 & 2.6 & 0.6 & 0.13 & 0.1 & 0.005 & 1.69 \\
\hline HE5 & 64.89 & 14.75 & 5.81 & 2.55 & 6.06 & 2.7 & 2.44 & 0.6 & 0.12 & 0.1 & 0.004 & 1.00 \\
\hline HE6 & 64.37 & 15.41 & 5.66 & 2.49 & 6.06 & 2.62 & 2.6 & 0.57 & 0.12 & 0.1 & 0.005 & 1.05 \\
\hline HE7 & 56.27 & 15.45 & 6.26 & 3.77 & 12.8 & 2.1 & 2.36 & 0.69 & 0.19 & 0.1 & 0.007 & 2.96 \\
\hline HE8 & 58.48 & 15.83 & 6.3 & 3.43 & 10.4 & 2.26 & 2.34 & 0.67 & 0.19 & 0.11 & 0.006 & 2.32 \\
\hline ME1 & 63.76 & 15.34 & 6.63 & 2.4 & 5.65 & 2.65 & 2.67 & 0.65 & 0.13 & 0.1 & 0.004 & 1.02 \\
\hline ME2 & 65.64 & 15.26 & 5.3 & 2.22 & 5.29 & 2.75 & 2.82 & 0.51 & 0.11 & 0.09 & 0.003 & 0.87 \\
\hline ME3 & 64.84 & 15.09 & 5.9 & 2.33 & 5.55 & 2.77 & 2.76 & 0.58 & 0.11 & 0.09 & 0.004 & 0.91 \\
\hline ME4 & 62.87 & 14.83 & 5.77 & 2.47 & 7.94 & 2.66 & 2.68 & 0.57 & 0.12 & 0.1 & 0.006 & 1.52 \\
\hline ME5 & 61.92 & 14.62 & 7.42 & 2.87 & 7.35 & 2.49 & 2.36 & 0.73 & 0.13 & 0.11 & 0.006 & 1.31 \\
\hline ME6 & 65.37 & 13.39 & 7.6 & 2.35 & 5.32 & 2.62 & 2.4 & 0.72 & 0.11 & 0.11 & 0.006 & 0.92 \\
\hline ME7 & 51.28 & 11.87 & 4.9 & 3.48 & 23.9 & 1.9 & 2.02 & 0.48 & 0.11 & 0.09 & 0.004 & 4.73 \\
\hline ME8 & 58.82 & 15.61 & 6.28 & 3.31 & 10.5 & 2.27 & 2.28 & 0.67 & 0.18 & 0.1 & 0.007 & 2.34 \\
\hline Average & 54.67 & 13.42 & 5.62 & 2.74 & 8.32 & 2.21 & 2.14 & 0.58 & 0.12 & 0.09 & 0.005 & 1.91 \\
\hline Mixed source & 61.60 & 15.35 & 4.81 & 3.50 & 5.71 & 3.59 & 1.33 & 0.42 & 0.05 & 0.09 & & \\
\hline AKT1 Terrace & 65.31 & 15.32 & 8.04 & 1.93 & 3.51 & 2.39 & 2.42 & 0.8 & 0.12 & 0.15 & 0.006 & 0.32 \\
\hline AKT2 & 64.43 & 18.39 & 7 & 1.82 & 2.49 & 1.86 & 3.17 & 0.58 & 0.11 & 0.15 & 0.006 & 0.29 \\
\hline AKT3 & 64.62 & 16.94 & 6.67 & 2.27 & 3.07 & 2.27 & 3.33 & 0.6 & 0.11 & 0.12 & 0.006 & 0.54 \\
\hline TET4 & 67.76 & 15.2 & 6.05 & 1.9 & 3.03 & 2.73 & 2.49 & 0.63 & 0.13 & 0.07 & 0.004 & 0.08 \\
\hline TET5 & 62.75 & 15.47 & 6.66 & 2.13 & 6.43 & 2.04 & 3.48 & 0.62 & 0.28 & 0.13 & 0.012 & 4.36 \\
\hline TET6 & 64.95 & 14.93 & 5.99 & 3.02 & 5.45 & 1.68 & 3.06 & 0.65 & 0.12 & 0.13 & 0.008 & 1.44 \\
\hline GMT3 & 65.97 & 16.68 & 6.22 & 2 & 2.83 & 2.77 & 2.66 & 0.64 & 0.12 & 0.12 & 0.004 & 0.04 \\
\hline GMT1 & 65.11 & 15.72 & 6.56 & 2.64 & 3.85 & 2.29 & 2.96 & 0.64 & 0.11 & 0.13 & 0.005 & 0.85 \\
\hline GMT2 & 64.98 & 15.05 & 6.51 & 2.67 & 4.56 & 2.56 & 2.8 & 0.64 & 0.12 & 0.11 & 0.004 & 0.82 \\
\hline HET1 & 63.58 & 16.36 & 7.43 & 2.36 & 4.34 & 2.43 & 2.48 & 0.73 & 0.13 & 0.15 & 0.004 & 0.42 \\
\hline HET2 & 64.71 & 15.57 & 7.19 & 2.08 & 4.71 & 2.31 & 2.5 & 0.74 & 0.11 & 0.09 & 0.005 & 0.81 \\
\hline MET3 & 56.63 & 16.91 & 8.27 & 3.16 & 9.91 & 2.11 & 1.92 & 0.72 & 0.19 & 0.18 & 0.003 & 1.54 \\
\hline MET4 & 61.92 & 17.22 & 7.86 & 2.66 & 4.25 & 2.51 & 2.33 & 0.73 & 0.13 & 0.37 & 0.013 & 0.45 \\
\hline MET5 & 57.85 & 17 & 10.2 & 3.21 & 5.67 & 2.89 & 1.91 & 0.93 & 0.14 & 0.15 & 0.002 & 0.13 \\
\hline Average & 58.85 & 14.98 & 6.65 & 2.23 & 4.21 & 2.17 & 2.47 & 0.64 & 0.12 & 0.13 & 0.006 & 0.86 \\
\hline Mixed source & 63.99 & 14.59 & 4.10 & 2.90 & 4.70 & 3.77 & 1.55 & 0.38 & 0.05 & 0.08 & & \\
\hline
\end{tabular}


Table 1 (Continued).

Çizelge 1 (Devam ediyor).

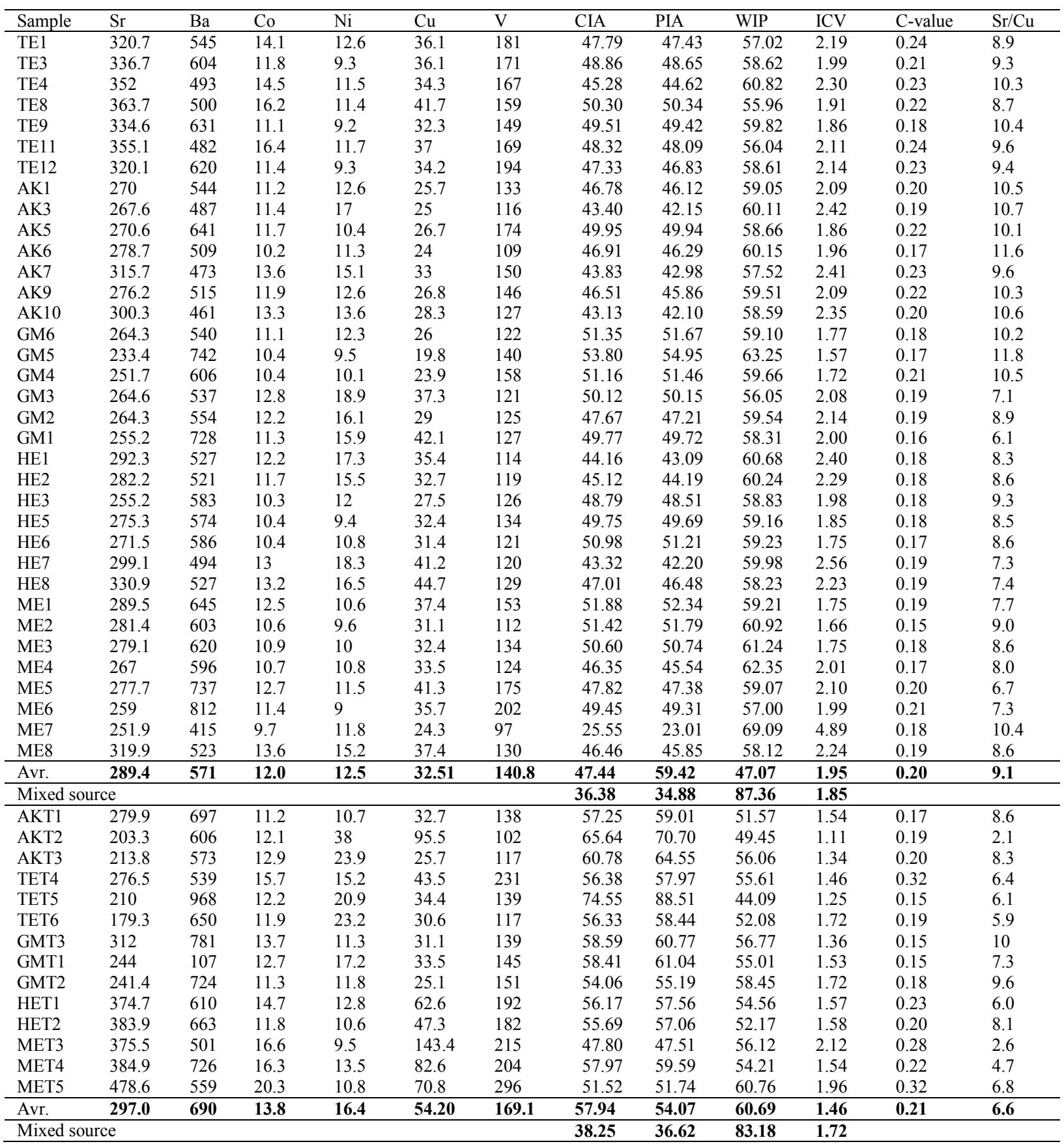


Table 1 (Continued).

Çizelge 1 (Devam ediyor).

\begin{tabular}{|c|c|c|c|c|c|c|c|c|c|c|c|c|c|c|c|c|}
\hline Sample & Th & $\mathrm{Sc}$ & $\mathrm{La}$ & $\mathrm{Ce}$ & $\mathrm{Pr}$ & $\mathrm{Nd}$ & $\mathrm{Sm}$ & $\mathrm{Eu}$ & Gd & $\mathrm{Tb}$ & Dy & Ho & $\mathrm{Er}$ & $\mathrm{Tm}$ & $\mathrm{Yb}$ & $\mathrm{Lu}$ \\
\hline TE1 & 4.8 & 16 & 19 & 33 & 3.6 & 14 & 2.8 & 0.8 & 2.7 & 0.4 & 2.6 & 0.5 & 1.6 & 0.3 & 1.8 & 0.3 \\
\hline TE3 & 4.9 & 16 & 18 & 32 & 3.4 & 14 & 2.5 & 0.7 & 2.6 & 0.4 & 2.5 & 0.5 & 1.4 & 0.3 & 1.6 & 0.3 \\
\hline TE4 & 4.3 & 16 & 18 & 33 & 3.7 & 15 & 3.1 & 0.8 & 2.9 & 0.5 & 2.9 & 0.6 & 1.8 & 0.3 & 1.8 & 0.3 \\
\hline TE5 & 1.5 & 8 & 11 & 14 & 1.8 & 7.5 & 1.4 & 0.4 & 1.5 & 0.2 & 1.5 & 0.3 & 0.9 & 0.1 & 0.9 & 0.1 \\
\hline TE8 & 4.7 & 17 & 18 & 34 & 3.8 & 15. & 3 & 0.8 & 3 & 0.5 & 2.6 & 0.6 & 1.7 & 0.3 & 1.8 & 0.3 \\
\hline TE9 & 4.8 & 14 & 18 & 33 & 3.6 & 15 & 3 & 0.8 & 2.8 & 0.4 & 2.6 & 0.6 & 1.7 & 0.3 & 1.7 & 0.3 \\
\hline TE11 & 4.5 & 17 & 19 & 36 & 3.9 & 15 & 3.1 & 0.8 & 3 & 0.5 & 3 & 0.6 & 1.7 & 0.3 & 1.8 & 0.3 \\
\hline TE12 & 5.1 & 15 & 19 & 35 & 3.8 & 15 & 3.1 & 0.7 & 2.7 & 0.4 & 2.7 & 0.5 & 1.7 & 0.3 & 1.7 & 0.3 \\
\hline AK1 & 5.8 & 13 & 21 & 40 & 4.4 & 17. & 3.2 & 0.8 & 3.2 & 0.5 & 3.0 & 0.7 & 2.0 & 0.3 & 2.0 & 0.3 \\
\hline AK3 & 6.1 & 13 & 20 & 38 & 4.1 & 16 & 3.2 & 0.7 & 3.1 & 0.5 & 3.3 & 0.7 & 2 & 0.3 & 2.0 & 0.3 \\
\hline AK5 & 5.6 & 14 & 19 & 34 & 3.9 & 15 & 3.0 & 0.7 & 2.9 & 0.5 & 2.9 & 0.6 & 1.8 & 0.3 & 1.8 & 0.3 \\
\hline AK6 & 5.1 & 12 & 18. & 36 & 3.9 & 15. & 3 & 0.7 & 2.9 & 0.5 & 2.9 & 0.6 & 1.8 & 0.3 & 1.8 & 0.3 \\
\hline AK7 & 4.8 & 15 & 19 & 34 & 3.9 & 15. & 3.2 & 0.8 & 3.1 & 0.5 & 2.8 & 0.6 & 1.9 & 0.3 & 1.7 & 0.2 \\
\hline AK9 & 5.0 & 14 & 18 & 35 & 3.8 & 15 & 3.2 & 0.8 & 3.2 & 0.6 & 3.1 & 0.7 & 2.0 & 0.3 & 2 & 0.3 \\
\hline AK10 & 5.2 & 14 & 18 & 36 & 3.8 & 14 & 2.9 & 0.7 & 3.0 & 0.5 & 2.8 & 0.5 & 1.7 & 0.3 & 1.8 & 0.3 \\
\hline GM6 & 5.9 & 13 & 20 & 37 & 4.1 & 16 & 3 & 0.7 & 3.1 & 0.5 & 3 & 0.7 & 1.9 & 0.3 & 2 & 0.3 \\
\hline GM5 & 11 & 13 & 31 & 58 & 6.1 & 23. & 4.5 & 0.9 & 4.2 & 0.7 & 3.9 & 0.8 & 2.6 & 0.4 & 2.7 & 0.4 \\
\hline GM4 & 5.4 & 13 & 20 & 35 & 39 & 15 & 2.9 & 0.7 & 2.9 & 0.5 & 2.9 & 0.6 & 1.8 & 0.2 & 1.8 & 0.2 \\
\hline GM3 & 7.8 & 15 & 25 & 48. & 5.1 & 20 & 4 & 0.9 & 3.8 & 0.7 & 3.9 & 0.8 & 2.5 & 0.4 & 2.4 & 0.4 \\
\hline GM2 & 7.6 & 15 & 24 & 45 & 4.9 & 19 & 3.8 & 0.8 & 3.7 & 0.7 & 3.7 & 0.8 & 2.3 & 0.4 & 2.5 & 0.4 \\
\hline GM1 & 7.5 & 13 & 24 & 46 & 4.9 & 19 & 3.8 & 0.8 & 3.6 & 0.6 & 3.5 & 0.7 & 2.3 & 0.3 & 2.3 & 0.3 \\
\hline HE1 & 7.1 & 14 & 21. & 41 & 4.5 & 17 & 3.5 & 0.8 & 3.4 & 0.6 & 3.5 & 0.7 & 2.2 & 0.3 & 2.2 & 0.4 \\
\hline HE2 & 7.2 & 14 & 23 & 41 & 4.3 & 16 & 3.1 & 0.7 & 3.4 & 0.6 & 3.3 & 0.8 & 2.3 & 0.3 & 2.4 & 0.4 \\
\hline HE3 & 7.2 & 13 & 20 & 39 & 4.1 & 16 & 3.1 & 0.7 & 3.2 & 0.5 & 3.2 & 0.8 & 1.9 & 0.3 & 2.1 & 0.3 \\
\hline HE5 & 5.7 & 14 & 18 & 35 & 3.8 & 15 & 2.9 & 0.8 & 3.0 & 0.5 & 3.2 & 0.7 & 1.9 & 0.3 & 1.9 & 0.3 \\
\hline HE6 & 7.4 & 14 & 21 & 39 & 4.2 & 16 & 3.2 & 0.7 & 3.3 & 0.5 & 3.1 & 0.7 & 2 & 0.3 & 2.1 & 0.3 \\
\hline HE7 & 7.1 & 15 & 22 & 43 & 4.6 & 18 & 3.4 & 0.8 & 3.5 & 0.6 & 3.3 & 0.7 & 2.1 & 0.3 & 2.2 & 0.4 \\
\hline HE8 & 7.2 & 15 & 21 & 39 & 4.3 & 16 & 3.2 & 0.8 & 3.4 & 0.5 & 3.3 & 0.7 & 2.1 & 0.3 & 2.2 & 0.3 \\
\hline ME1 & 7.0 & 14 & 21 & 41 & 4.4 & 16 & 3.2 & 0.8 & 3.3 & 0.5 & 3.2 & 0.7 & 2.1 & 0.3 & 2.2 & 0.4 \\
\hline ME2 & 6.5 & 12 & 20 & 36 & 3.9 & 15 & 3 & 0.7 & 2.9 & 0.5 & 2.9 & 0.6 & 1.8 & 0.3 & 1.9 & 0.3 \\
\hline ME3 & 6.7 & 13 & 21 & 38 & 4.1 & 15 & 3.0 & 0.7 & 3.1 & 0.5 & 2.9 & 0.7 & 1.9 & 0.3 & 2 & 0.3 \\
\hline ME4 & 7.5 & 13 & 23 & 38 & 4.2 & 16 & 3.1 & 0.7 & 3.2 & 0.5 & 3.1 & 0.7 & 1.8 & 0.3 & 2.0 & 0.3 \\
\hline ME5 & 7.7 & 16 & 25 & 45 & 4.9 & 18 & 3.6 & 0.9 & 3.6 & 0.6 & 3.5 & 0.8 & 2.3 & 0.4 & 2.4 & 0.4 \\
\hline ME6 & 6.7 & 13 & 23 & 40 & 4.3 & 16 & 3.2 & 0.8 & 3.2 & 0.5 & 3.1 & 0.7 & 1.9 & 0.3 & 2.1 & 0.3 \\
\hline ME7 & 4.9 & 9 & 15 & 27 & 3.0 & 12 & 2.2 & 0.5 & 2.4 & 0.4 & 2.2 & 0.5 & 1.3 & 0.2 & 1.3 & 0.2 \\
\hline ME8 & 6.9 & 14 & 23 & 42 & 4.5 & 16 & 3.6 & 0.8 & 3.5 & 0.6 & 3.5 & 0.7 & 2.2 & 0.3 & 2.2 & 0.4 \\
\hline TET4 & 5.5 & 14 & 21 & 39 & 4.0 & 15 & 3.2 & 0.8 & 3 & 0.5 & 3.2 & 0.7 & 1.9 & 0.3 & 2.0 & 0.3 \\
\hline TET5 & 10.4 & 12 & 24 & 42 & 4.9 & 19 & 3.8 & 0.9 & 3.9 & 0.6 & 3.8 & 0.8 & 2.4 & 0.4 & 2.2 & 0.4 \\
\hline TET6 & 8.1 & 14 & 39 & 68 & 7.2 & 27 & 4.7 & 0.9 & 4.7 & 0.8 & 4.4 & 1 & 3 & 0.5 & 3.1 & 0.5 \\
\hline AKT1 & 5.8 & 15 & 20 & 38 & 4.2 & 16 & 3.3 & 0.8 & 3.3 & 0.5 & 3.2 & 0.7 & 2.1 & 0.3 & 2.0 & 0.3 \\
\hline AKT2 & 7.7 & 17 & 31 & 57 & 6.1 & 23 & 4.3 & 0.8 & 4.3 & 0.7 & 4 & 0.8 & 2.5 & 0.8 & 2.4 & 0.4 \\
\hline AKT3 & 15.4 & 15 & 26 & 53 & 5.7 & 21 & 4.4 & 0.8 & 4.3 & 0.7 & 4.2 & 0.9 & 2.4 & 0.4 & 2.4 & 0.4 \\
\hline GMT3 & 6.3 & 15 & 22 & 40 & 4.4 & 17 & 3.5 & 0.8 & 3.4 & 0.5 & 3.3 & 0.7 & 2.1 & 0.3 & 2.2 & 0.3 \\
\hline GMT1 & 7.9 & 13 & 2 & 44 & 4.2 & 16 & 3.2 & 0.7 & 3.1 & 0.5 & 3.2 & 0.6 & 1.9 & 0.3 & 2.0 & 0.3 \\
\hline GMT2 & 8.3 & 13 & 22 & 43 & 4.5 & 17. & 3.3 & 0.8 & 3.2 & 0.5 & 3.2 & 0.7 & 1.9 & 0.3 & 1.9 & 0.3 \\
\hline HET1 & 8.2 & 17 & 24 & 45 & 4.8 & 18 & 3.8 & 0.9 & 3.6 & 0.6 & 3.5 & 0.8 & 2.3 & 0.4 & 2.3 & 0.4 \\
\hline HET2 & 6.6 & 16 & 19 & 38 & 3.9 & 15 & 3.2 & 0.8 & 3.2 & 0.5 & 3.1 & 0.7 & 2.0 & 0.3 & 2.1 & 0.3 \\
\hline MET3 & 4.8 & 17 & 18 & 34 & 3.7 & 14 & 2.8 & 0.9 & 2.9 & 0.5 & 2.7 & 0.5 & 1.7 & 0.3 & 1.7 & 0.3 \\
\hline MET4 & 7.0 & 19 & 21 & 42 & 4.1 & 16 & 3.1 & 0.9 & 3.1 & 0.5 & 3.1 & 0.7 & 2 & 0.3 & 2 & 0.3 \\
\hline MET5 & 5.4 & 21 & 17 & 33 & 3.7 & 15 & 3.1 & 0.9 & 3.4 & 0.5 & 3.3 & 0.7 & 1.9 & 0.3 & 2.2 & 0.3 \\
\hline
\end{tabular}


Table 2. Correlation coefficients ( $r$ ) from the correlation matrix obtained using geochemical data from the analyzed samples ( $n=35$ for bed, $n=14$ for terrace sediments).

Çizelge 2. Analiz edilen örneklerin kimyasal verilerinin kullanılarak elde edilen korelasyon katsayıları (r) (yatak için 35, taraça için 14 örnek).

\begin{tabular}{|c|c|c|c|c|c|c|c|c|c|c|c|c|c|}
\hline & $\mathrm{SiO}_{2}$ & $\mathrm{Al}_{2} \mathrm{O}_{3}$ & $\mathrm{~F}_{2} \mathrm{O}_{3}$ & $\mathrm{MgO}$ & $\mathrm{CaO}$ & $\mathrm{Na} 2 \mathrm{O}$ & $\mathrm{K}_{2} \mathrm{O}$ & $\mathrm{TiO}_{2}$ & $\mathrm{P}_{2} \mathrm{O}_{5}$ & $\mathrm{MnO}$ & TOC & CIA & PIA \\
\hline $\mathrm{SiO}_{2}$ & 1.000 & & & & & & & & & & & & \\
\hline $\mathrm{Al}_{2} \mathrm{O}_{3}$ & .296 & 1.000 & & & & & & & & & & & \\
\hline $\mathrm{Fe}_{2} \mathrm{O}_{3}$ & .200 & $.364^{(*)}$ & 1.000 & & & & & & & & & & \\
\hline $\mathrm{MgO}$ & $-.923^{(* *)}$ & -.234 & -.105 & 1.000 & & & & & & & & & \\
\hline $\mathrm{CaO}$ & $-.949^{(* *)}$ & $-.477^{(* *)}$ & -.328 & $.876^{(* *)}$ & 1.000 & & & & & & & & \\
\hline $\mathrm{Na}_{2} \mathrm{O}$ & $.941^{(* *)}$ & $.456^{(* *)}$ & .283 & $-.879^{(* *)}$ & $-.932^{(* *)}$ & 1.000 & & & & & & & \\
\hline $\mathrm{K}_{2} \mathrm{O}$ & $.797^{(* *)}$ & .267 & -.056 & $-.792^{(* *)}$ & $-.814^{(* *)}$ & $.751^{(* *)}$ & 1.000 & & & & & & \\
\hline $\mathrm{Ti}_{2} \mathrm{O}$ & -.051 & .310 & $.926^{(* *)}$ & .172 & -.088 & .034 & -.227 & 1.000 & & & & & \\
\hline $\mathrm{P}_{2} \mathrm{O}_{5}$ & $-.593^{(*)}$ & .194 & .290 & $.646^{(* *)}$ & $.487^{(* *)}$ & $-.496^{(* *)}$ & $-.448^{(*)}$ & $.521^{(* *)}$ & 1.000 & & & & \\
\hline $\mathrm{MnO}$ & -.186 & .184 & $.735^{(* *)}$ & .299 & .068 & -.106 & -.306 & $.799^{(* *)}$ & $.502^{(* *)}$ & 1.000 & & & \\
\hline TOC & $-.926^{(*)}$ & $-.493^{(* *)}$ & $-.407^{(*)}$ & $.828^{(* *)}$ & $.920^{(* *)}$ & $-.931^{(* *)}$ & $-.616^{(* *)}$ & -.169 & $.462^{(* *)}$ & -.004 & 1.000 & & \\
\hline CIA & $.838^{(* *)}$ & $.649^{(+*)}$ & $.360^{(*)}$ & $-.764^{(* *)}$ & $-.949^{(* *)}$ & $.832^{(* *)}$ & $.759^{(* *)}$ & .159 & $-.358^{(*)}$ & .002 & $-.854^{(* *)}$ & 1.000 & \\
\hline PIA & $.838^{(* *)}$ & $.654^{(* *)}$ & $.375^{(*)}$ & $-.770^{(* *)}$ & $-.949^{(* *)}$ & $.837^{(* *)}$ & $.743^{(* *)}$ & .169 & $-.352^{(*)}$ & .012 & $-.862^{(* *)}$ & $.998^{(* *)}$ & 1.000 \\
\hline
\end{tabular}

${ }^{*}$ Correlation is significant at the 0.05 level ${ }^{* *}$ Correlation is significant at the 0.01 level

\begin{tabular}{|c|c|c|c|c|c|c|c|c|c|c|c|c|c|}
\hline & $\mathrm{SiO}_{2}$ & $\mathrm{Al}_{2} \mathrm{O}_{3}$ & $\mathrm{~F}_{2} \mathrm{O}_{3}$ & $\mathrm{MgO}$ & $\mathrm{CaO}$ & $\mathrm{Na} 2 \mathrm{O}$ & $\mathrm{K}_{2} \mathrm{O}$ & $\mathrm{TiO}_{2}$ & $\mathrm{P}_{2} \mathrm{O}_{5}$ & $\mathrm{MnO}$ & TOC & $\mathrm{CIA}$ & PIA \\
\hline $\mathrm{SiO}_{2}$ & 1.000 & & & & & & & & & & & & \\
\hline $\mathrm{Al}_{2} \mathrm{O}_{3}$ & -.055 & 1.000 & & & & & & & & & & & \\
\hline $\mathrm{Fe}_{2} \mathrm{O}_{3}$ & -.279 & .495 & 1.000 & & & & & & & & & & \\
\hline $\mathrm{MgO}$ & -.470 & .015 & .276 & 1.000 & & & & & & & & & \\
\hline $\mathrm{CaO}$ & $-.727^{(* *)}$ & -.468 & .134 & $.616^{(*)}$ & 1.000 & & & & & & & & \\
\hline $\mathrm{Na}_{2} \mathrm{O}$ & .407 & .327 & .288 & .143 & -.156 & 1.000 & & & & & & & \\
\hline $\mathrm{K}_{2} \mathrm{O}$ & .213 & -.260 & $-.748^{(*)}$ & -.427 & -.354 & -.504 & 1.000 & & & & & & \\
\hline $\mathrm{Ti}_{2} \mathrm{O}$ & -.024 & .241 & $.761^{(* *)}$ & .356 & .210 & $.626^{(*)}$ & $-.899^{(*)}$ & 1.000 & & & & & \\
\hline $\mathrm{P}_{2} \mathrm{O}_{5}$ & $-.537^{(*)}$ & -.054 & .149 & .274 & $.614^{(*)}$ & .221 & -.460 & .253 & 1.000 & & & & \\
\hline $\mathrm{MnO}$ & $-.577\left(^{*}\right)$ & .487 & $.644^{(*)}$ & .337 & .152 & -.145 & -.484 & .355 & .294 & 1.000 & & & \\
\hline TOC & -.530 & $-.662^{(* *)}$ & -.235 & .338 & $.662^{(* *)}$ & $-.666^{(*)}$ & .224 & -.381 & .115 & .027 & 1.000 & & \\
\hline CIA & .235 & 196 & -.354 & $-.753^{(*)}$ & $-.653^{\left({ }^{*}\right)}$ & -.270 & $.660^{(*)}$ & $-.591^{(*)}$ & -.348 & -.101 & -.165 & 1.000 & \\
\hline PIA & .213 & .138 & -.380 & $-.744^{(* *)}$ & $-.600^{(*)}$ & -.266 & $.664^{(* *)}$ & $-.595^{(*)}$ & -.296 & -.125 & -.121 & $.996^{(* *)}$ & 1.000 \\
\hline
\end{tabular}




\section{DISCUSSIONS}

\section{Mixed source composition}

Previous research (Cullers, 1994, 1995; Jian et al., 2013) have exhibited that finegrained sediments are more likely to have mineralogical and chemical composition similar to their source rocks compositions and so they may be better indicators of provenance. Major, trace and rare earth elements data of different potential sources are collected and used to model the notional proportions of end members for Harşit bed and terrace sediments. The first end-member is Carboniferous granodiorite (Gümüşhane pluton, Topuz et al., 2010), the second end-member is Lower Jurassic gabbro (Gökcedere pluton, Karslı et al., 2017), and the third end-member is Upper Cretaceous felsic tuffs (Eyuboğlu, 2015). These three end-members can provide a wide range of composition for identifying likely source end-member proportions (Table 3, Fig. 3A, B).

Using the granodiorite, gabbro and felsic tuffs as the potential source contributors, it is possible to quantitatively model their proportions. A mixture of $3 \%$ granodiorite $(\mathrm{Gd})+50 \%$ gabbro $(\mathrm{Gb})+47 \%$ felsic tuff $(\mathrm{TF})$ is suggested as the ideal modeling source composition for Harşit bed sediments, and $5 \%$ granodiorite $+37 \%$ gabbro + $58 \%$ felsic tuff for terrace sediments, because they are almost similar chondritenormalized REE pattern (Fig. 4A, B). In addition, the average $E u / E u^{*},(G d / Y b)_{N}$, $\mathrm{La} / \mathrm{Th}$ and $(\mathrm{La} / \mathrm{Yb})_{\mathrm{N}}$ ratios $(0.79,1.15,3.40$ and 6.20 , respectively) of the first mixture model of $3 \% \mathrm{Gd}+50 \% \mathrm{~Gb}+47 \%$ TF are nearly similar to those of bed sediments $\left(0.74,1.29,3.47\right.$ and 7.02 , respectively). Eu/Eu* $(\mathrm{Gd} / \mathrm{Yb})_{\mathrm{N}}, \mathrm{La} / \mathrm{Th}$ and $(\mathrm{La} / \mathrm{Yb})_{\mathrm{N}}$ ratios $(0.74,1.16,3.06$ and 7.10 , respectively) of the second mixture model of $5 \% \mathrm{Gd}+$ $37 \% \mathrm{~Gb}+58 \% \mathrm{TF}$ are nearly similar to those of terrace sediments $0.74,1.30,3.20$, 7.10 respectively) (Table 3 ). The average $\mathrm{Co} / \mathrm{Th}$ and $\mathrm{Th} / \mathrm{Sc}$ ratios of the first and second mixed models $(14.16,10,54$ and $1.80,2.22$, respectively) are higher than those of the bed and terrace sediments $(2.11,2.00$ and $0.40,0.50$, respectively) (Table 3). The average $\mathrm{Eu} / \mathrm{Eu}^{*},(\mathrm{Gd} / \mathrm{Yb})_{\mathrm{N}}, \mathrm{La} / \mathrm{Th}$ and $(\mathrm{La} / \mathrm{Yb})_{\mathrm{N}}$ ratios of the studied sediments coincide with the 1:1 line, whereas, the average $\mathrm{Co} / \mathrm{Th}$ and $\mathrm{Th} / \mathrm{Sc}$ ratios of both sediments are somewhat far from the 1:1 line (Fig. 4C, D), and this case can be described by the enrichment of mafic fragments and decreased of quartz during sedimentary processes. Additionally, the bed sediments have lower average $\mathrm{SiO}_{2}$, $\mathrm{Al}_{2} \mathrm{O}_{3}, \mathrm{MgO}$ and $\mathrm{Na}_{2} \mathrm{O}$ concentrations than that of the suggested modeled mixed 
source rocks, but higher concentrations of $\mathrm{Fe}_{2} \mathrm{O}_{3}, \mathrm{CaO}, \mathrm{K}_{2} \mathrm{O}, \mathrm{TiO}_{2}$ and $\mathrm{P}_{2} \mathrm{O}_{5}$ (Fig. 5A). The terrace sediments have lower average have $\mathrm{SiO}_{2}, \mathrm{MgO}, \mathrm{CaO}, \mathrm{Na}_{2} \mathrm{O}$ and $\mathrm{MnO}$ concentration than that of the suggested mixed source rocks, but higher concentrations of $\mathrm{Al}_{2} \mathrm{O}_{3}, \mathrm{Fe}_{2} \mathrm{O}_{3}, \mathrm{~K}_{2} \mathrm{O}, \mathrm{TiO}_{2}$ and $\mathrm{P}_{2} \mathrm{O}_{5}$ (Table 3, Fig. 5B).

Table 3. Major, trace and rare earth elements of source end members, mixing source and average of the studied sediments.

Çizelge 3. Incelenen örneklerin, karışık kaynak ve kaynak üyelerinin ana, iz ve nadir toprak element ortalamaları.

\begin{tabular}{|c|c|c|c|c|c|c|c|}
\hline \multirow[t]{2}{*}{ Element } & \multicolumn{3}{|c|}{$\begin{array}{c}\text { End Members } \\
\text { Granodiorite Gabbro Felsic tuff }\end{array}$} & \multirow{2}{*}{$\begin{array}{l}\begin{array}{l}\text { Mixed source } \\
\text { model for bed } \\
\quad \text { sediments }\end{array} \\
3 \% \\
\text { Gd+50\%Gb+4 } \\
7 \% \mathrm{FT}\end{array}$} & \multirow{2}{*}{$\begin{array}{l}\text { Mixed source model } \\
\text { for terrace } \\
\text { sediments } \\
5 \% \\
\text { Gd+37\%Gb+58\%F } \\
T\end{array}$} & \multicolumn{2}{|c|}{ Studied sediments } \\
\hline & Gd. & Gb. & FT & & & Bed sediments & $\begin{array}{l}\text { Terrace } \\
\text { sediment } \\
\text { s }\end{array}$ \\
\hline $\mathrm{SiO}_{2}$ & 64.77 & 52.11 & 71.50 & 61.6 & 63.99 & 54.67 & 58.85 \\
\hline $\mathrm{Al}_{2} \mathrm{O}_{3}$ & 15.26 & 18.46 & 12.06 & 15.35 & 14.59 & 13.42 & 14.98 \\
\hline $\mathrm{Fe}_{2} \mathrm{O}_{3}$ & 5.10 & 7.70 & 1.71 & 4.81 & 4.1 & 5.62 & 6.65 \\
\hline $\mathrm{MgO}$ & 2.76 & 5.86 & 1.03 & 3.5 & 2.9 & 2.74 & 2.23 \\
\hline $\mathrm{CaO}$ & 3.55 & 9.70 & 1.60 & 5.71 & 4.7 & 8.32 & 4.21 \\
\hline $\mathrm{Na}_{2} \mathrm{O}$ & 2.99 & 2.83 & 4.44 & 3.59 & 3.77 & 2.21 & 2.17 \\
\hline $\mathrm{K}_{2} \mathrm{O}$ & 3.11 & 0.57 & 2.03 & 1.33 & 1.55 & 2.14 & 2.47 \\
\hline $\mathrm{TiO}_{2}$ & 0.48 & 0.58 & 0.25 & 0.42 & 0.38 & 0.58 & 0.64 \\
\hline $\mathrm{P}_{2} \mathrm{O}_{5}$ & 0.10 & 0.05 & 0.05 & 0.05 & 0.05 & 0.12 & 0.12 \\
\hline $\mathrm{MnO}$ & 0.08 & 0.14 & 0.03 & 0.09 & 0.08 & 0.09 & 0.13 \\
\hline Th & 13.4 & 1.24 & 16.53 & 8.79 & 11.28 & 6.1 & 7.7 \\
\hline Sc & 14.2 & 33.79 & 5.15 & 19.74 & 15.05 & 13.9 & 15.6 \\
\hline Co & 13.7 & 23.34 & 2.34 & 13.18 & 9.68 & 11.9 & 13.8 \\
\hline La & 22.10 & 4.65 & 34.59 & 19.25 & 24.19 & 20.36 & 23.25 \\
\hline $\mathrm{Ce}$ & 41.70 & 10.33 & 61.55 & 35.35 & 43.79 & 37.66 & 43.87 \\
\hline $\mathrm{Pr}$ & 4.80 & 1.37 & 6.38 & 3.83 & 4.65 & 4.10 & 4.66 \\
\hline $\mathrm{Nd}$ & 17.70 & 6.65 & 21.70 & 14.05 & 16.51 & 15.74 & 17.84 \\
\hline Sm & 3.50 & 2.00 & 3.95 & 2.96 & 3.28 & 3.14 & 3.55 \\
\hline $\mathrm{Eu}$ & 0.80 & 0.66 & 0.71 & 0.69 & 0.69 & 0.75 & 0.84 \\
\hline Gd & 3.40 & 2.77 & 3.53 & 3.15 & 3.27 & 3.11 & 3.52 \\
\hline $\mathrm{Tb}$ & 0.60 & 0.47 & 0.60 & 0.54 & 0.56 & 0.51 & 0.57 \\
\hline Dy & 3.30 & 3.28 & 3.48 & 3.38 & 3.41 & 3.05 & 3.43 \\
\hline $\mathrm{Ho}$ & 0.70 & 0.71 & 0.76 & 0.73 & 0.74 & 0.65 & 0.74 \\
\hline $\mathrm{Er}$ & 2.10 & 2.08 & 2.34 & 2.20 & 2.25 & 1.92 & 2.15 \\
\hline $\mathrm{Tm}$ & 0.30 & 0.31 & 0.37 & 0.34 & 0.35 & 0.29 & 0.33 \\
\hline $\mathrm{Yb}$ & 2.10 & 2.04 & 2.45 & 2.24 & 2.31 & 1.97 & 2.19 \\
\hline Lu & 0.30 & 0.32 & 0.39 & 0.35 & 0.36 & 0.31 & 0.35 \\
\hline $\mathrm{Th} / \mathrm{Sc}$ & 0.95 & 0.04 & 0.58 & 1.80 & 2.22 & 0.40 & 0.50 \\
\hline $\mathrm{La} / \mathrm{Th}$ & 1.66 & 4.7 & 9.98 & 3.40 & 3.06 & 3.47 & 3.20 \\
\hline $\mathrm{Co} / \mathrm{Th}$ & 1.06 & 28.12 & 1.18 & 14.16 & 10.54 & 2.11 & 2.00 \\
\hline $\mathrm{Eu} / \mathrm{Eu}^{*}$ & 0.71 & 0.99 & 3.72 & 0.79 & 0.74 & 0.74 & 0.74 \\
\hline $\mathrm{LaN}_{N} / \mathrm{Yb}_{\mathrm{N}}$ & 6.98 & 2.59 & 2.13 & 6.20 & 7.10 & 7.02 & 7.10 \\
\hline $\mathrm{Gd}_{N} / \mathrm{Yb}_{\mathrm{N}}$ & 1.28 & 1.11 & 0.14 & 1.15 & 1.16 & 1.29 & 1.30 \\
\hline
\end{tabular}


In substance, REEs distributions, Eu/Eu*, $(\mathrm{Gd} / \mathrm{Yb})_{\mathrm{N}}, \mathrm{La} / \mathrm{Th}$ and $(\mathrm{La} / \mathrm{Yb})_{N}$ ratios of the bed and terrace sediments show that they were derived from mixed sources ( $A$ mixture of $3 \%$ granodiorite $+50 \%$ gabbro $+47 \%$ felsic tuff and a mixture of $5 \%$ granodiorite $+37 \%$ gabbro $+58 \%$ felsic tuff, respectively) .

\section{Weathering intensity}

Major element chemistry is best practicable for defining the weathering degree of source sediment to interpret the weathering intensity. Different indices such as the Chemical Index of Alteration (CIA, Nesbitt and Young, 1982), the Plagioclase Index of Alteration (PIA, Fedo et al., 1995), the Weathering Index (WIP, Parker, 1970) and the Index of Composition Variability (ICV, Cullers and Podkovyrov, 2002), were improved to determine the level of weathering. The CIA is a chemical representative to evaluate the degree of switch of minerals to clay minerals. The CIA values in unaltered rocks are between 45 and 55 for granite, granodiorite and rhyolite, and between 30 and 45 for fresh gabbro and basalt. The CIA values are $\sim 10$ for unweathered clinopyroxene, $\sim 30$ for unweathered hornblende, and $\sim 50$ for unweathered feldspars (Nesbitt and Young, 1982; Zhou et al., 2017). The value is higher for sediment and sedimentary rocks, which are formed of complex mineralogical mixtures (carbonates, phyllosilicates and phosphates). In aluminumrich phyllosilicates the CIA values range from 75 to 100 (100 for chlorite and kaolinite, 80 for illite and smectite, and 75 for muscovite) (Nesbitt and Young, 1982). Sediments with high WIP and ICV values may have been derived from immature sediments and characterize first-cycle deposits; sediments with low WIP and ICV values may have been derived from mature sediments (Kamp and Leake, 1985; Wang et al., 2014; Saydam Eker and Demirkol Kiliç, 2018). 

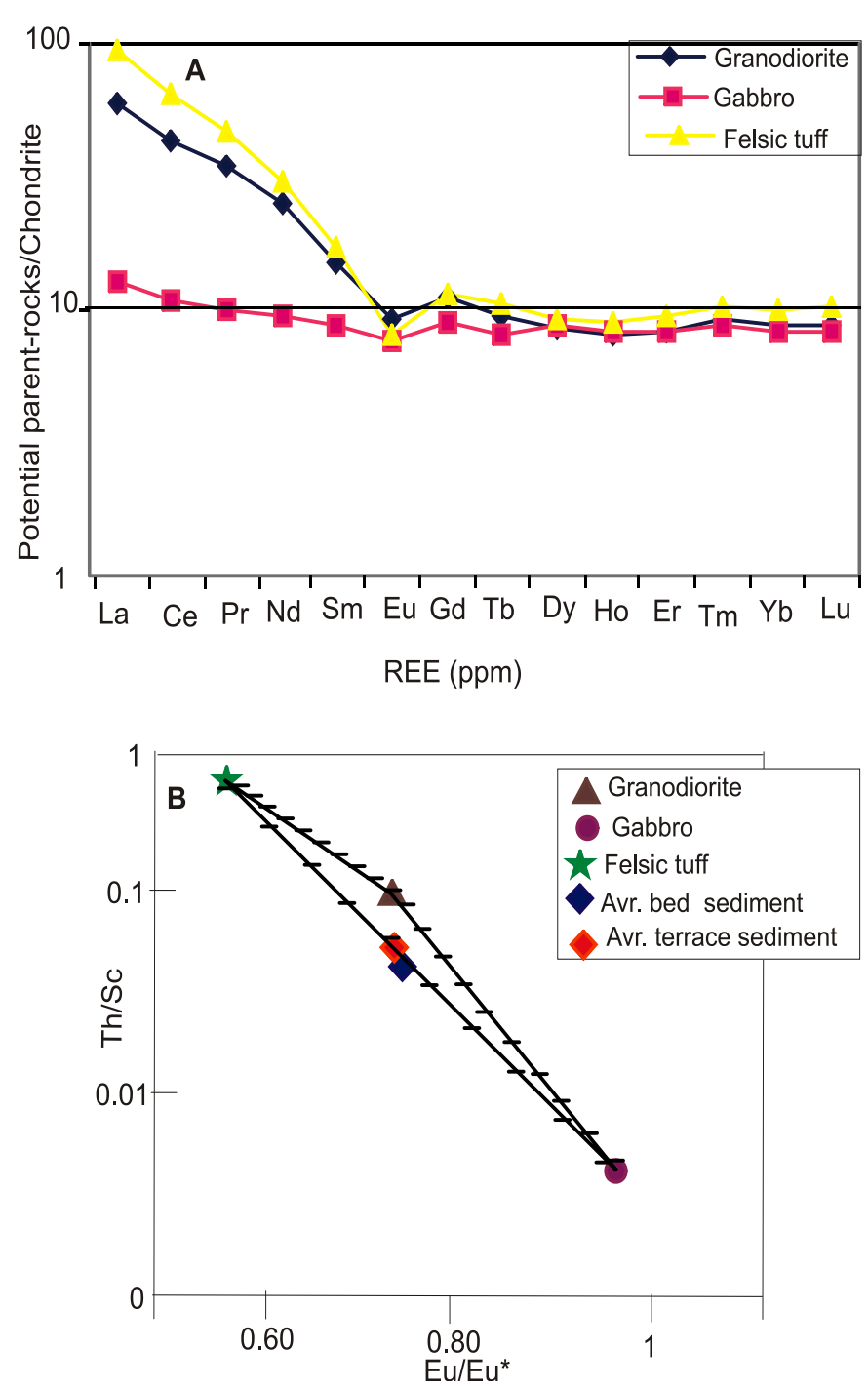

Figure 3. A. Chondrite-normalized REE patterns of Potential parent-rock end members for the bed and terrace sediments, B. Plot of Th/Sc versus Eu/Eu* for the sediments. Mixing curves are divided into $10 \%$ increments, with a mixture of $3 \%$ granodiorite, $\% 50$ gabbro, $47 \%$ felsic tuff for bed sediments and $5 \%$ granodiorite, \% 37 gabbro, $58 \%$ felsic tuff for terrace sediments.

Şekil 3. A. Kanal ve taraça sedimentleri için potansiyel kaynak kayaçların kondritle normalize edilmiş REE diyagramı, $B$. Sedimentler için $\mathrm{Th} / \mathrm{Sc}$ ve $E u / E u^{*}$ diyagramı. Karışım eğrisi \%10 aralıklarla bölünmüştür. Kanal sediment için \%3 granodiyorit, \%50 gabro, \%47 felsik tüf ve taraça sedimenti için \%5 granodiyorit, \%37 gabro, \%58 felsik tüf karışımı alınmıştır. 

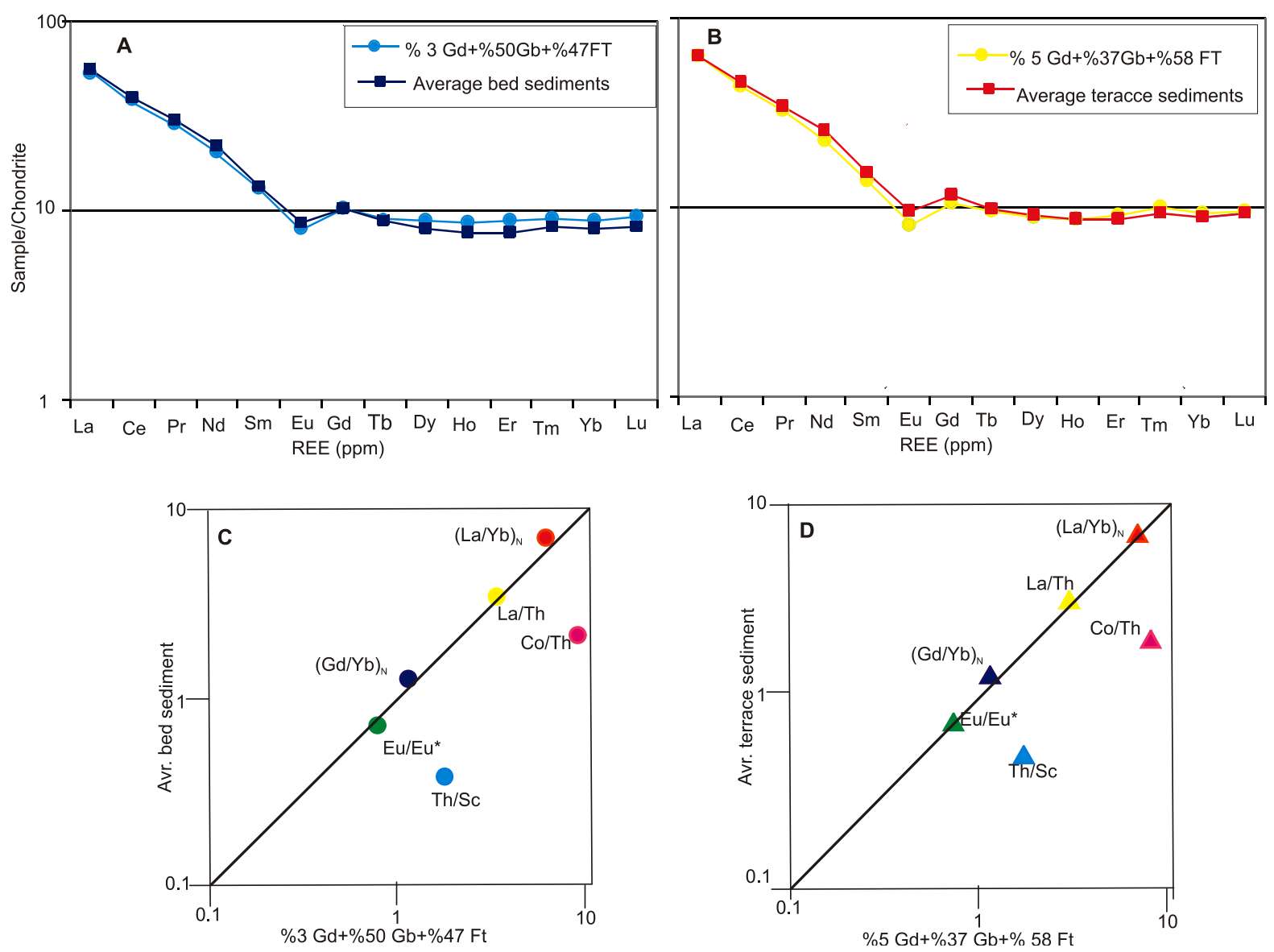

Figure 4. Comparison between the modeled mixed source and the average studied sediments composition. Chondrite-normalized REE patterns for studied sediments (A, B) and trace elemental ratios (C, D).

Şekil 4. Modellenmiş karışık kaynak ve çalışılan sediment bileşenlerinin karşılaştııılması. Çalışılan sedimentler için kondritle normalize edilmiş $\operatorname{NTE}(A, B)$ ve iz element oranları $(C, D)$.

The Chemical Index of Alteration (CIA), described as [CIA= $100 \times \mathrm{Al}_{2} \mathrm{O}_{3} /\left(\mathrm{Al}_{2} \mathrm{O}_{3}+\mathrm{CaO} *+\mathrm{Na}_{2} \mathrm{O}+\mathrm{K}_{2} \mathrm{O}\right.$ ) (Nesbitt and Young, 1982)], the Plagioclase Index of Alteration [PIA $=\left(\mathrm{Al}_{2} \mathrm{O}_{3}-\mathrm{K}_{2} \mathrm{O}\right) /\left(\mathrm{Al}_{2} \mathrm{O}_{3}-\mathrm{K}_{2} \mathrm{O}\right)+\left(\mathrm{CaO}^{*}+\mathrm{Na}_{2} \mathrm{O}\right) \times 100$, Fedo et al., 1995], the Weathering Index $\left[\mathrm{WIP}=100 \times\left(\mathrm{CaO} / 0.7+2 \quad \mathrm{Na}_{2} \mathrm{O} / 0.35+2\right.\right.$ $\mathrm{K}_{2} \mathrm{O} / 0.25+\mathrm{MgO} / 0.9$, Parker, 1970] and the Index of Compositional Variability [ICV = $\mathrm{Fe}_{2} \mathrm{O}_{3}+\mathrm{K}_{2} \mathrm{O}+\mathrm{Na}_{2} \mathrm{O}+\mathrm{CaO}+\mathrm{MgO}+\mathrm{TiO}_{2} / \mathrm{Al}_{2} \mathrm{O}_{3}$, Cullers and Podkovyrov, 2002] (in molecular proportion) is used to appraise the weathering intensity of rocks. $\mathrm{CaO}^{*}$ represents the amount of $\mathrm{CaO}$ added in the silicate fraction of the rock by expecting Ca incorporated in apatite and carbonate (Zhou et al., 2017). 

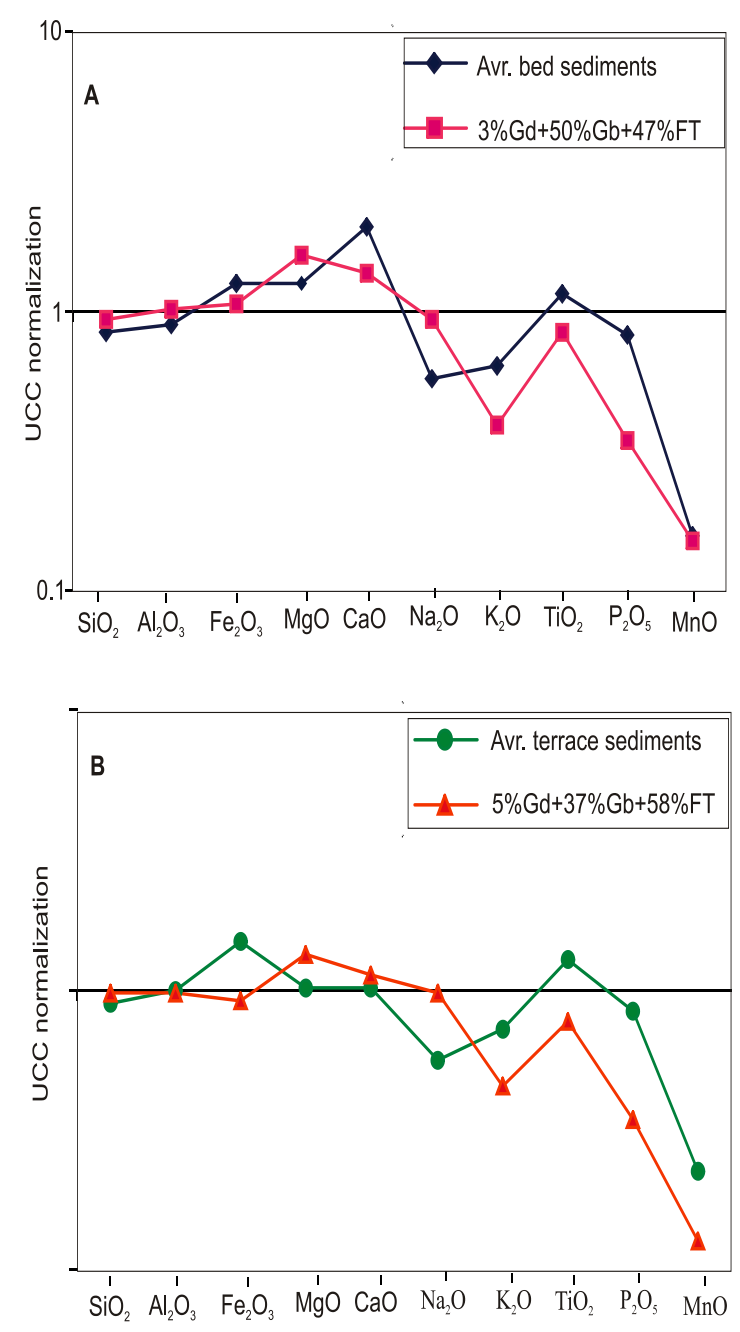

Figure 5. UCC-normalized (Taylor and McLennan, 1985) major elements of the modeled mixed sources and the average of the studied sediments composition. A: bed sediments, $B$ : terrace sediments.

Şekil 5. Incelenen sediment ve karışık kaynak kayaçlarının ortalama ana elementlerinin ÜKK ile normalize edilmiş örümcek diyagramı (Taylor ve McLennan, 1985). A: Kanal sedimentleri, B: Taraça sedimentleri.

The possible source rocks of the Harşit sediments are the Upper Cretaceous felsic tuffs, the Early Jurassic gabbro and the Carboniferous granodiorite. The gabbro is unweathered as shown by its very low CIA, PIA values (29, 28, respectively) and high WIP, ICV values $(98,2.4$, respectively). The felsic tuff and granodiorite are lowly weathered as shown by their low CIA, PIA values (44, 40 and 42, 38, respectively) and medium - high WIP, ICV values (70, 82 and 1.4, 1.7, respectively). The mixed source rocks for Harşit bed and terrace sediments have low CIA and PIA values (36, 38 and 35, 37, respectively), and high WIP and ICV values (84, 82 and 1.9, 1.8) (Table 1). The CIA and PIA values of terrace sediments are higher than those of bed sediments. The CIA values calculated accordingly vary from 26 to 54 for the bed sediments and 48 to 75 for the terrace sediments, showing a mild weathering history for bed sediments and a mild to moderate weathering history for terrace sediments. The CIA value is used to reflect the progressive alteration of potassium feldspar and plagioclase to clay minerals during the weathering of rocks (Nesbitt and Young, 1982; 
Zhou et al., 2017). The PIA index is used to evaluate the degree of weathering of plagioclase in rocks. The PIA values of the bed sediments are between 23 and 55 and terrace sediments are between 47 and 89 , indicating the bed sediments contain better preserved plagioclase than the terrace sediments. The high positive correlation of PIA and CIA values for the bed sediments ( $r=0.998)$, and the terrace sediments $(r=0.996)$ are required to show the minimal weathering in the sediments. At the same time, PIA values are higher than CIA values in both sediments, because differential rates of K-feldspar and plagioclase weathering are mostly observed in bed rocks and its sediments (Goldich, 1938; White et al., 2001); Zhou et al., 2017). At the beginning of magma cooling, plagioclase crystallizes and forms chains of physically linked phenocrysts in crystalline rocks (Bryon et al., 1995). Conversely, the latter nucleation and growth of K-feldspar are limited to interstices of this crystalline framework and are more physically divided (Zhou, et al., 2017). In addition, experimental dissolution of plagioclase indicates that $\mathrm{Na}$-rich plagioclase is more slowly dissolved than $\mathrm{Ca}$-rich plagioclase (Blum and Stillings, 1995). The WIP and ICV values of the terrace sediments are lower than those of the bed sediments (average of 54, 1.46 and 59, 1.95 , respectively).

The weathering intensity of the source of various sediments has been evaluated using the A-CN-K ternary diagram (Fig. 6). The diagram indicates kinetically and empirically presumable weathering vectors for different rock types and minerals, and strengthening for graphical interpretation of proportional chemical changes (Nesbitt and Young, 1984; Nesbitt and Wilson, 1992; Zhou et al., 2017). The general weathering vector for feldspar decomposition in different parent rocks is need to be parallel to the A-CN axis, but the certain vector direction is a function of the relative proportion of K-feldspar and plagioclase, their incongruent or congruent resolution, and the rate of preservation of aluminous weathering products (Babechuk et al., 2015). On the A-CN-K ternary diagram, the granodiorite, the gabbro, the felsic tuffs, the mixed source rocks for bed and terrace sediments and the analyzed samples exhibit a linear array along weathering trend, falling on the A-CN axis (Fig. 6). The Harşit bed sediments are ranged between 40 and 50 values (except two samples), indicating that the samples are lowly weathered. The terrace sediments are ranged between 50 and 65 values along weathering line (Fig. 6), indicating that the samples are lowly to moderately weathered (Taylor and McLennan, 1985). In addition, intensive chemical weathering may direct to increase the $\mathrm{Rb} / \mathrm{Sr}$ ratio, therefore ratios 
$>1$ are indicators of intense weathering (Armstrong-Altrin et al., 2019; McLennan et al., 1993). The bed and terrace sediments have low $\mathrm{Rb} / \mathrm{Sr}$ ratios, the average $\mathrm{Rb} / \mathrm{Sr}$ ratio was calculated as 0.23 for the bed sediments, and as 0.31 for the terrace sediments (Saydam Eker, 2017). This result supports the low chemical weathering for bed sediments and low to moderate chemical weathering for terrace sediments.

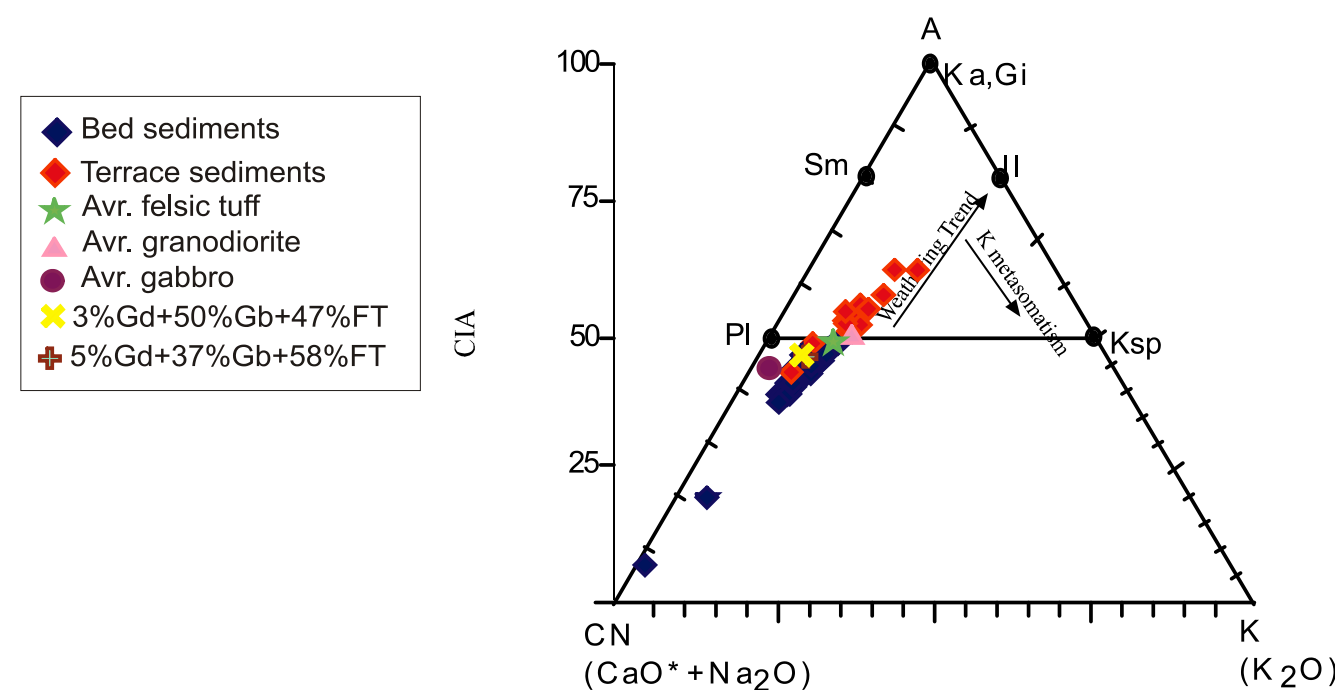

Figure 6. A-CN-K ternary diagram of the studied sediments, potential parent-rock end members (granodiorite, gabbro, felsic tuff) and modeled mixed source. $A=\mathrm{Al}_{2} \mathrm{O}_{3} ; \mathrm{CN}=\left(\mathrm{CaO}{ }^{*}+\mathrm{N}_{2} \mathrm{O}\right.$ ); $\mathrm{K}=\mathrm{K}_{2} \mathrm{O}$ (in molar proportions). $\mathrm{ClA}=$ Chemical Index of Alteration. Mineral composition: $\mathrm{Pl}=$ Plagioclase; $\mathrm{Ksp}=\mathrm{K}-$ feldspar; Ka= Kaolinite; Gi= Gibbsite; Sm= Smectite; II= Ilite (Nesbitt and Young, 1984).

Şekil 6. Incelenen sedimentlerin, potansiyel kaynak kayaların (Granodiyorit, gabro, felsik tüf) ve modellenmiş karışık kaynak kayaların A-CN-K üçgen diyagramı. $A=A_{2} \mathrm{O}_{3} ; \mathrm{CN}=\left(\mathrm{CaO}{ }^{*}+\mathrm{N}_{2} \mathrm{O}\right) ; \mathrm{K}=\mathrm{K}_{2} \mathrm{O}$ (molar oranlar). $C I A=K i m y a s a l$ alterasyon indeksi. Mineral bileşen: $P I=$ plajioklaz; $K s p=K$-feldispat; Ka= Kaolinit; Gi= Jipsit; Sm= Smektit, II= Illit (Nesbitt ve Young, 1984).

\section{Paleoclimate conditions}

Time differences in climate can be a significant factor for the weathering of source area for the bed and terrace sediments. Since the weathering index values of the terrace sediments are higher, the climate was likely more humid in the study area a few hundred years ago. In addition, the composition and distribution of some trace and major elements in sedimentary rocks may disclose the paleoclimatic variations (Hu et al., 2017; Vd'ačný' et al., 2019). Cao et al. (2012) suggested that Mn, Fe, Cr, $\mathrm{Co}, \mathrm{Ni}$ and $\mathrm{V}$ are relatively enriched under humid conditions. Contrarily, $\mathrm{Ca}, \mathrm{Mg}, \mathrm{K}$, $\mathrm{Na}, \mathrm{Sr}$ and $\mathrm{Ba}$ are relatively enriched under arid conditions. Some research successfully performed the C-value as a proxy for climate changes (Zhao et al., 2007; Fu et al., 2016; Wang et al., 2017; Vd’ačný et al., 2019). 
The C-value is calculated by the following formula:

$\mathrm{C}-$ value $=\Sigma(\mathrm{Fe}+\mathrm{Mn}+\mathrm{Cr}+\mathrm{Ni}+\mathrm{V}+\mathrm{Co}) / \Sigma(\mathrm{Ca}+\mathrm{Mg}+\mathrm{Na}+\mathrm{K}+\mathrm{Sr}+\mathrm{Ba})$

The C-values for the bed sediments range from 0.15 to 0.24 (on average 0.20 ), for the terrace sediments, from 0.15 to 0.32 (on average 0.21 ) (Table 1). This reflects a generally arid to semiarid climate during the depositional period of bed and terrace sediments, but the climate conditions in a few hundreds years ago (depositional period of terrace sediments) is relatively more humid than present.

In addition, the $\mathrm{Sr} / \mathrm{Cu}$ ratio index has been used for paleoclimatic studies (Meng et al., 2012; Jia et al., 2013; Hu et al., 2017). In general, low Sr/Cu ratios show a warm humid climate, whereas high $\mathrm{Sr} / \mathrm{Cu}$ ratios indicate a hot climate (Jia et al., 2013). Lerman (1978) suggested that Sr/Cu ratios between 1.3 and 5.0 are determinative of a warm humid climate, while $\mathrm{Sr} / \mathrm{Cu}$ ratio $>5.0$ exhibits a hot arid climate. The $\mathrm{Sr} / \mathrm{Cu}$ ratios of the bed sediments range from 6.1 to 11.6 (on average 9.1), terrace sediments range from 2.1 to 10.6 (on average 6.6) (Table 1). This distribution of $\mathrm{Sr} / \mathrm{Cu}$ ratios indicate that an arid to semiarid climate conditions dominated during the depositional period of bed sediments and an arid to semi-moist climate conditions dominated during the depositional period of terrace sediments.

\section{Quantification of mass transfer}

To quantify mass transfer during weathering of the probable source rocks (granodiorite, gabbro and felsic tuffs), the tau (T) mass-transport model (Brimhall and Dietrich, 1987; Anderson et al., 2002; Zhou et al., 2017) was performed to mass balance calculations. Titanium is regarded as the immobile index element for $\tau$ calculation. In this model, the concentrations (C) of elements (j) in the source rocks (p), relative to that of an immobile index element (i), are used as a normalization to determine the mass changes in the progressively altered rocks using the formula (Zhou et al., 2017).

$W: T(i, j)=\left[\left(C_{j, w}\right) /\left(C_{j, p}\right)\right] /\left[\left(C_{i, w}\right) /\left(C_{i, p}\right)\right]-1$ 
When $\tau_{j}=0$, element is immobile during weathering with respect to the source rocks, indicating no gain and loss of mass. When $\tau_{j}=-1$, the element is completely removed during weathering. The results calculated for selected elements are listed in Table 4. During weathering of the mixed source rocks, $\mathrm{Na}$ exhibits the greatest loss ( $\tau_{\mathrm{Na}}$ from -0.86 to -0.92 ) for the bed sediments, followed by Si ( $T_{s i}$ from -0.80 to -0.88 ), K (TK from -0.75 to -0.88$), \mathrm{Al}, \mathrm{Mg}$ ( $\mathrm{T}_{\mathrm{Al}}, \mathrm{Mg}$ from -0.77 to -0.86$)$, $\mathrm{Mn}$ ( $\mathrm{T}_{\mathrm{Mn}}$ from -0.67 to -0.73 ), Fe ( $T_{\mathrm{Fe}}$ from -0.63 to -0.68 ), Ca (TCa from -0.47 to -0.79 , except two samples) and $P$ ( $T_{P}$ from -0.39 to -0.67 ). Two samples exhibits unusual high positive values of $T_{\mathrm{Ca}}=$ 0.44 - 2.88 (Table 4, Fig. 7A), which may include the occasional autogenic deposition. More rapidly dissolution of Ca-rich plagioclase than Na-rich plagioclase supports autogenic mineral deposition. The $\mathrm{Na}, \mathrm{Si}, \mathrm{K}, \mathrm{Mg}$ and $\mathrm{Al}$ are highly depleted, $\mathrm{Mn}$ and Fe are moderately depleted, $\mathrm{P}$ and $\mathrm{Ca}$ are moderately - slightly depleted, but $\mathrm{Ca}$ is sometimes enriched in the bed sediment samples (Fig. 7A). For the terrace sediments, Na exhibits the greatest loss ( $\tau_{\mathrm{Na}}$ from -0.86 to -0.90 ), followed by Si ( $\mathrm{TSi}_{\mathrm{Si}}$ from -0.80 to -0.89$), \mathrm{Mg}$ ( $T_{\mathrm{Mg}}$ from -0.79 to -0.89$), \mathrm{K}$ ( $T_{\mathrm{K}}$ from -0.65 to -0.89 ), Al ( $T_{\mathrm{Al}}$ from -0.74 to -0.85$)$, $\mathrm{Ca}$ ( $\mathrm{T}_{\mathrm{Ca}}$ from -0.60 to -0.87 ), Fe ( $\mathrm{T}_{\mathrm{Fe}}$ from -0.62 to -0.71 ), $\mathrm{Mn}$ ( $T_{M n}$ from -0.47 to -0.81 ) and $P$ (TP from -0.48 to -0.69 ) (Table 4). $\mathrm{Na}, \mathrm{Si}, \mathrm{Mg}$ and $\mathrm{Al}$ are highly depleted, K, Ca and Fe are highly - moderately depleted, Mn is slightly highly depleted and $\mathrm{P}$ is slightly - moderately depleted in the terrace sediment samples (Fig. 7B). The average of $\mathrm{Si}, \mathrm{Al}, \mathrm{Na}, \mathrm{K}$ and $\mathrm{Mn}$ shows more loss in bed sediments than terrace sediments, the average of $\mathrm{Fe}, \mathrm{Mg}, \mathrm{Ca}$ and $\mathrm{P}$ shows less loss in bed sediments than terrace sediments (Table 4, Fig. 7C). The $\mathrm{T}_{\mathrm{si}}$ values were lost in both sediments during weathering of silicates, only in part kept in the secondary clay minerals. The important depletion of $\mathrm{Na}$ in the bed and terrace sediments likely reflects that weathering occurred in humid climatic conditions. The rate of K-feldspar weathering is very restricted, because it has a lower resolution than plagioclase (Holland and Turekian, 2010). Therefore, K-feldspars were with lower loss than plagioclase in the studied sediments. 
Although the PIA and CIA values of terrace sediments are higher than those of bed sediments, quantification of mass transfer of the elements is generally higher in the bed sediments. It may be because river water causes silicate hydrolysis and transports soluble weathering products away from reaction sites.
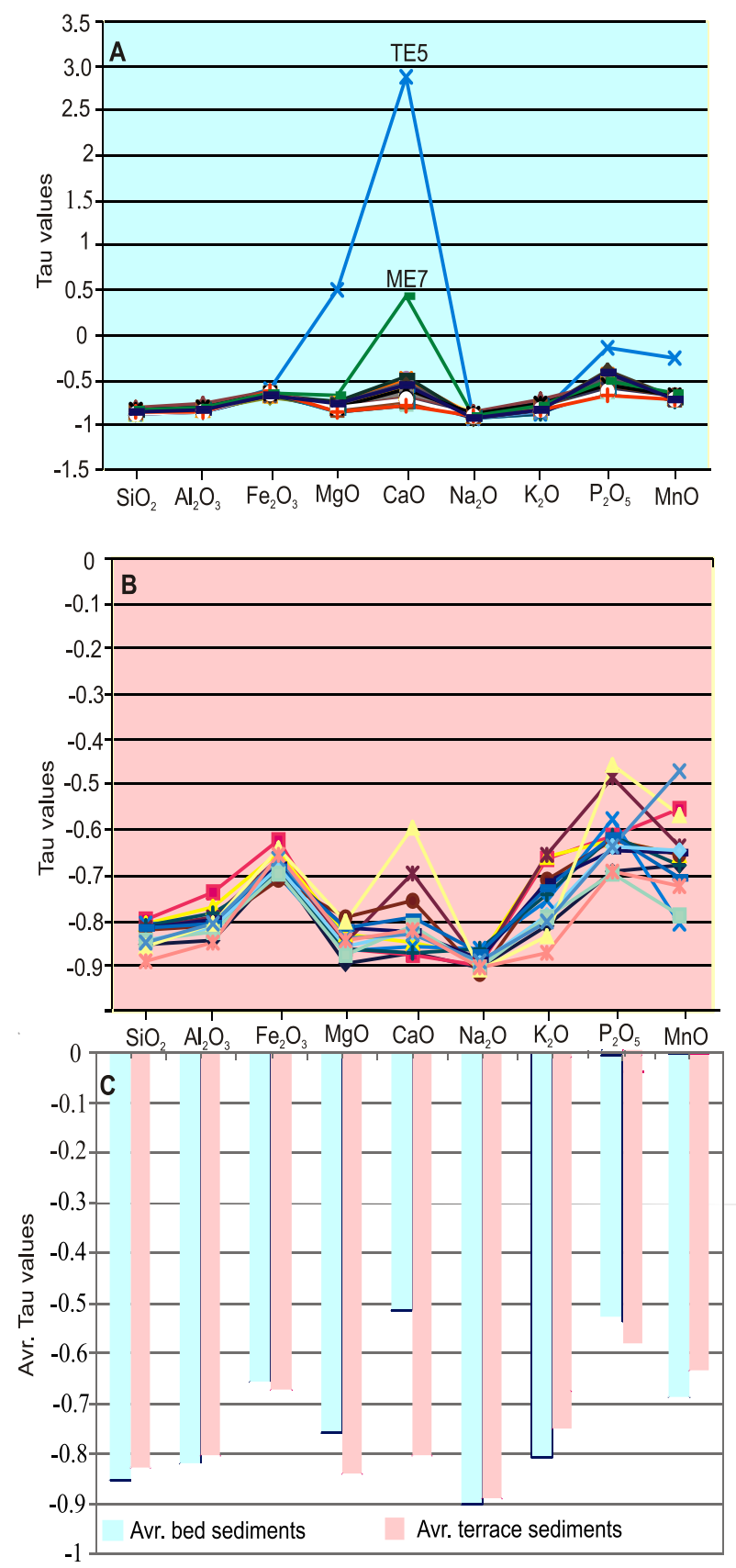

Figure 7. Distributions of $T$ values for major elements of the studied samples. A: bed sediments, B: terrace sediments, C: average of bed and terrace sediments.

Şekil 7. Incelenen örneklerin major element değerlerinin dağılımı. A: Kanal sediment, $B$ : Taraça sediment, C: Kanal ve taraça sediment ortalaması. 


\section{CONCLUSIONS}

1. The Harşit bed sediment samples have higher average of $\mathrm{MgO}, \mathrm{CaO}$ and $\mathrm{Na}_{2} \mathrm{O}$ concentrations than samples from terrace sediments, but lower concentrations of $\mathrm{SiO}_{2}$, $\mathrm{Al}_{2} \mathrm{O}_{3}, \mathrm{Fe}_{2} \mathrm{O}_{3}, \mathrm{~K}_{2} \mathrm{O}, \mathrm{TiO}_{2}$ and $\mathrm{MnO}$.

2. The $\mathrm{Eu} / \mathrm{Eu}^{*}, \mathrm{La} / \mathrm{Th},(\mathrm{Gd} / \mathrm{Yb})_{N},(\mathrm{La} / \mathrm{Yb})$ ratios of the average bed and terrace sediments are closely reproduced by mixing source rocks showed in the bed sediments in following properties: $3 \%$ granodiorite $+50 \%$ gabbro $+47 \%$ felsic tuff and in the terrace sediments in following properties: $5 \%$ granodiorite $+37 \%$ gabbro $+58 \%$ felsic tuff.

3. The CIA and PIA values of bed sediments are lower than those of terrace sediments. The CIA values calculated accordingly vary from 26 to 54 for bed sediments, and 48 to 75 for terrace sediments, indicating a low weathering history for bed sediments and a low to moderate weathering history for terrace sediments.

4. The distribution of $\mathrm{C}$-values and $\mathrm{Sr} / \mathrm{Cu}$ ratios reflect a generally arid to semiarid climate conditions during the depositional period of bed sediments and an arid to semi-moist climate conditions during the depositional period of terrace sediments.

5- During weathering of the mixed source rocks, Na exhibits the greatest loss for bed and terrace sediments, $\mathrm{P}$ exhibits the lowest loss. The average of $\mathrm{Si}, \mathrm{Al}, \mathrm{Na}, \mathrm{K}$ and $\mathrm{Mn}$ shows more loss in bed sediments than terrace sediments, the average of $\mathrm{Fe}, \mathrm{Mg}, \mathrm{Ca}$ and $\mathrm{P}$ shows less loss in bed sediments than terrace sediments.

Finally, the PIA and CIA values of terrace sediments are higher than those of bed sediments, whereas quantification of mass transfer of the elements is generally higher in the bed sediments. It may be because river water causes silicate hydrolysis and transports soluble weathering products away from reaction sites.

\section{ACKNOWLEDGEMENTS}

The author gratefully acknowledges M. Tekin Yürür and Gülbanu Tekbulut, from the Hacettepe University, Yerbilimleri Uygulama ve Araştırma Merkezi, for editorial handling. I would like to thank two anonymous reviewers for their contributions to the manuscript. 


\section{REFERENCES}

Alexander, P.O. and Gibbson, I.L., 1977. Rare earth abundances in Deccan trap basalts. Lithos, 10, 143-147.

Anderson, S.P., Dietrich, W.E. and Brimhall, G.H., 2002. Weathering profiles, massbalance analysis, and rates of solute loss: linkages between weathering and erosion in a small, steep catchment. Geological Society of America Bulletin, 14, 1143-1158.

Armstrong-Altrin, J.S., Lee, Y.I., Kasper-Zubillaga, J.J. and Trejo-Ramírez, E., 2017. Mineralogy and geochemistry of sands along the Manzanillo and El Carrizal beach areas, southern Mexico: implications for palaeoweathering, provenance, and tectonic setting. Geological Journal, 52, 559-582.

Armstrong-Altrin, J.S., Botello, A.V., Villanueva, S.F. and Soto, L.A., 2019. Geochemistry of surface sediments from the north western Gulf of Mexico: implications for provenance and heavy metal contamination. Geological Quarterly, 63, 522-538.

Armstrong-Altrin, J.S., Ramos-Vázquez, M.A., Zavala-León, A.C. and Montiel-García, P.C., 2018. Provenance discrimination between Atasta and Alvarado beach sands, western Gulf of Mexico, Mexico: Constraints from detrital zircon chemistry and U-Pb geochronology. Geological Journal, 53, 2824-2848.

Aslan, Z., Arslan, M., Temizel, İ. and Kaygusuz, A., 2014. K-Ar dating, whole-rock and Sr$\mathrm{Nd}$ isotope geochemistry of calc-alkaline volcanic rocks around the Gümüşhane area: implications for post-collisional volcanism in the Eastern Pontides, Northeast Turkey. Mineralogy and Petrology, 108, 245-267.

Babechuk, M.G., Widdowson, M., Murphy, M. and Kamber, B.S., 2015. A combined Y/Ho, high field strength element (HFSE) and Nd isotope perspective on basalt weathering, Deccan traps, India. Chemical Geology, 396, 25-41.

Bhatia M.R., 1983. Plate tectonics and geochemical composition of sandstones. Journal of Geology, 91, 611-627.

Blum, A.E. and Stillings, L.L., 1995. Chemical weathering of feldspars. Chemical Weathering Rates of Silicate Minerals. Min. Soc. Am. Rev. Min., 31, 291-351.

Brimhall, G.H. and Dietrich, W.E., 1987. Constitutive mass balance relations between chemical composition, volume, density, porosity, and strain in metasomatic hydrochemical systems: results on weathering and pedogenesis. Geochimica et Cosmochimica Acta, 51, 567-587.

Bryon, D.N., Atherton, M.P. and Hunter, R.H., 1995. The interpretation of granitic textures from serial thin sectioning, image analysis and three-dimensional reconstruction. Mineralogical Magazine, 59, 203-211. 
Cao, J., Wu, M., Chen, Y., Hu, K., Bian, L., Wang, L. and Zhang, Y., 2012. Trace and rare earth element geochemistry of Jurassic mudstones in the northern Qaidam Basin, northwest China. Chemie der Erde, 72, 245-252.

Climate-Data-Org., 2020. Annual climate in Gümüşhane, Turkey. https://tr.climatedata.org/asya/tuerkiye/guemueshane-665/, accessed on 15 April 2020.

Condie, K.C., 1991. Another look at the rare earth elements in shales. Geochimica et Cosmochimica Acta, 35, 2527-2531.

Cullers, R. L., 1994. The controls on the major and trace element variation of shales, siltstones of Pennsylvanian-Permian age from uplifted continental blocks in Colorado to platform sediment in Kansas, USA. Geochimica et Cosmochimica Acta, 5, 49554972.

Cullers, R. L., 1995. The controls on the major and trace element evolution of shales, siltstones and sandstones of Ordovician to Tertiary age in the wet Mountains region, Colorado, USA. Chemical Geology, 123, 107-131.

Cullers, R.L. and Podkovyrov, N., 2002. The source and origin of terrigenous sedimentary rocks in the Mesoproterozoic Ui group, southeastern Russia. Precambrian Research, 117, 157-183.

Çoğulu, E., 1975. Gümüşhane ve Rize Granitik Plutonlarının Mukayeseli Petrojeolojik ve Jeokronolojik Etüdü. PhD, İstanbul Technical University, İstanbul, Turkey (Unpublished).

Dokuz, A., 2011. A slab detachment and delamination model for the generation of Carboniferous high-potassium I-type magmatism in the Eastern Pontides, NE Turkey: The Köse composite pluton. Gondwana Research, 19, 926-944.

Eyuboglu, Y., 2015. Petrogenesis and U-Pb zircon chronology of felsic tuffs interbedded with turbidites (Eastern Pontides Orogenic Belt, NE Turkey): Implications for Mesozoic geodynamic evolution of the eastern Mediterranean region and accumulation rates of turbidite sequences. Gondwana Research, 212-215, 74-92.

Fedo, C.M., Nesbitt, H.W. and Young, G.M., 1995. Unraveling the effects of potassium metasomatism in sedimentary rock sand paleosols, with implications for paleoweathering conditions and provenance. Geology, 23, 921-924.

Fedo, C.M., Eriksson, K.A. and Krogstad, E.J., 1996. Geochemistry of shales from the Archean ( 3.0 Ga) Buhwa Greenstone Belt, Zimbabwe: implications for provenance and source-area weathering. Geochimica et Cosmochimica Acta, 60, 1751-1764. 
Fedo, C.M., Young, G.M. and Nesbitt, H.W., 1997. Paleoclimatic control on the composition of the Paleoproterozoic Serpent Formation, Huronian Supergroup, Canada: a greenhouse to icehouse transition. Precambrian Research, 86, 201-223.

Fu X, Wang J, Chen W, Feng X, Wang D, Song C, Zeng S (2016) Elemental geochemistry of the early Jurassic black shales in the Qiangtang Basin, eastern Tethys: constraints for palaeoenvironment conditions. Geological Journal 51: 443-454

Goldich, S.S., 1938. A study in rock-weathering. Journal of Geology, 46, 17-58.

Güven, I.H., 1993. 1/100.000 scaled geological map series of Turkey. 57-60, MTA Publication, Ankara.

Holland, H.D. and Turekian, K.K., 2010. Geochemistry of Earth Surface Systems. A Derivative of the Treatise on Geochemistry, In: Holland HD, Turekian KK (eds), Academic Press, pp. 227-230.

Hu, J., Li, Q., Song, C., Wang, S. and Shen, B., 2017. Geochemical characteristics of the Permian sedimentary rocks from Qiangtang Basin: constraints for paleoenvironment and paleoclimate. Terrestrial, Atmospheric and Oceanic Sciences, 28, 271-282.

Jia, J., Liu, Z., Bechtel, A., Strobl, S.A.I. and Sun, P. 2013. Tectonic and climate control of oil shale deposition in the Upper Cretaceous Qingshankou Formation (Songliao Basin, NE China). International Journal of Earth Sciences, 102, 1717-1734.

Jian, X., Guan, P., Zhang, W. and Feng, F., 2013. Geochemistry of Mesozoic and Cenozoic Sediments in the Northern Qaidam Basin, Northeastern Tibetan Plateau: Implications for Provenance and Weathering. Chemical Geology, 360-361, 74-88.

Johnsson, M.J., 1993. The system controlling the composition of clastic sediments. Processes controlling the composition of clastic sediments, In: Johnsson M.J., Basu A. (eds), Geological Society of America Special Paper, 284, 1-19.

Kamp, P.C. and Leake, B.E., 1985. Petrography and geochemistry of feldspathic and mafic sediments of the northeastern Pacific margin. Transaction of the Royal Society of Edinburgh. Earth Sciences, 76, 411-449.

Kaotekwar, A.B., Ahmad, S.M., Satyanarayanan, M. and Krishana, A.K., 2019. Geochemical investigations in bulk and clay size fractions from lower Krishna river sediments, southern India: implications of elemental fractionation during weathering, transportation and deposition. Geosciences Journal, 23, 951-960.

Karslı, O., Dokuz, A. and Kandemir, R., 2017. Zircon Lu-Hf isotope systematics and U-Pb geochronology, whole-rock Sr-Nd isotopes and geochemistry of the early Jurassic Gokcedere pluton, Sakarya Zone-NE Turkey: a magmatic response to roll-back of the 
Paleo-Tethyan oceanic lithosphere. Contributions to Mineralogy and Petrology, 172, 31.

Kaygusuz, A., Arslan, M., Siebel, W. and Şen, C., 2011. Geochemical and Sr-Nd isotopic characteristics of post-collisional calc-alkaline volcanics in the eastern Pontides (NE Turkey). Turkish Journal of Earth Sciences, 20, 137-159.

Kaygusuz, A., Arslan, M., Siebel,W., Sipahi, F. and İlbeyli, N., 2012. Geochronological evidence and tectonic significance of Carboniferous magmatism in the southwest Trabzon area, eastern Pontides, Turkey. International Geology Review, 1776-1800.

Kaygusuz, A., Arslan, M., Sipahi, F. and Temizel, İ., 2016. U-Pb zircon chronology and petrogenesis of Carboniferous plutons in the northern part of the Eastern Pontides, NE Turkey: Constraints for Paleozoic magmatism and geodynamic evolution. Gondwana Research, 39, 327-346.

Kessarkar, P.M., Rao, V.P. and Shynu, R., 2010. The nature and distribution of particulate matter in the Mandovi estuary, central West coast of India. Estuarine, Coastal and Shelf Science, 3, 30-44.

Lerman, A., 1978. Lakes: Chemistry, Geology, Physics. Springer-Verlag, New York.

Martin, J.M. and Meybeck, M., 1979. Elemental mass-balance of material carried by major world rivers. Marine Chemistry, 7, 173-206.

McLennan, S.M., 1989. Rare earth elements in sedimentary rocks; influence of provenance and sedimentary processes. Reviews in Mineralogy Geochemistry, 21, 169-200.

McLennan, S.M. and Taylor, S.R., 1991. Sedimentary rocks and crustal evolution: tectonic setting and secular trends. Journal of Geology, 99, 1-21.

McLennan, S.M., Hemming, S., McDaniel, D.K. and Hanson, G.N., 1993. Geochemical approaches to sedimentation, provenance, and tectonics. Processes Controlling the Composition of Clastic Sediments, In: Johnsson, M.J. and Basu, A. (eds), Geol. Soc. Am. Spec. Pub., 284, pp. 21-40.

Meng, Q., Liu, Z., Bruch, A.A., Liu, R. and Hu, F., 2012. Palaeoclimatic evolution during Eocene and its influence on oil shale mineralisation, Fushun basin, China. Journal of Asian Earth Science, 45, 95-105.

Milliman, J.D. and Meade, R.H., 1983. World-wide delivery of river sediment to the oceans. Journal of Geology, 91, 1-21.

Nesbitt, H.W., 1979. Mobility and fractionation of REE during weathering of granodiorite. Nature, 279, 206-210. 
Nesbitt, H.W., Markovics, G. and Price, R.C., 1980. Chemical processes affecting alkalis and alkaline earths during continental weathering. Geochim. Cosmochim. Acta, 44, 1659-1666.

Nesbitt, H.W. and Wilson, R.E., 1992. Recent chemical weathering of basalts. American Journal of Science, 292, 740-777.

Nesbitt, H.W. and Young, G. M., 1982. Early Proterozoic climates and plate motions inferred from major element chemistry of lutites. Nature, 299, 715-717.

Nesbitt, H.W. and Young, G.M., 1984. Predictions of some weathering trends of plutonic and volcanic rocks based on thermodynamic and kinetic considerations. Geochim. Cosmo. Acta, 48, 1523-1534.

Parker, A., 1970. An index of weathering for silicate rocks. Geological Magazine, 107, 501504.

Piper, D.Z.,1974. Rare earth elements in the sedimentary cycle: a summary. Chemical Geology, 14, 285-304.

Roddaz, M., Said, A., Guillot, S., Antoine, P.O., Montel, J-M., Martin, F. and Darrozes, J., 2011. Provenance of Cenozoic sedimentary rocks from the Sulaiman Fold and Thrust Belt, Pakistan: Implications for the Palaeogeography of the Indus Drainage System. Journal of Geology Society, 168, 499-516.

Roser, B.P. and Korsch, R.J., 1986. Determination of tectonic setting of sandstonemudstone suites using $\mathrm{SiO} 2$ content and K2O/Na2O ratio. J. Geol, 94, 635-650.

Saydam, Eker, C. and Korkmaz, S., 2011. Mineralogy and whole rock geochemistry of late Cretaceous sandstones from the eastern Pontides (NE Turkey). Neues Jahrbuch Für Mineralogy-Abhandlungen, 188 (3), 235-256.

Saydam, Eker, Ç., 2017. Geochemical and isotopic characteristics of stream and terrace sediments of the Harsit Stream, NE Turkey. Geochemistry: Exploration, Environment, Analysis, 17 (4), 279-296.

Saydam, Eker, Ç. and Demirkol, Kiliç, E., 2018. Geochemistry of Çoruh River bed sediments in NE Turkey: Implications in weathering-sedimentary cycle, provenance, and metal pollution. Geochemistry International, 56 (6), 579-600.

Schoenborn, W.A. and Fedo, C.M., 2011. Provenance and paleoweathering reconstruction of the Neoproterozoic Johnnie Formation, southeastern California. Chemical Geology, $285,231-255$.

Sensarma, S., Rajamani, V. and Tripathi, J.K., 2008. Petrography and geochemical characteristics of the sediments of the small River Hemavati, southern India: implications for provenance and weathering processes. Sedimentary Geology, 205, $111-125$. 
Taylor, S.R. and McLennan, S.M., 1985. The Continental Crust: Its Composition and Evolution. Oxford, Blackwell Scientific Publishing.

Topuz, G., Altherr, R., Kalt, A., Satır, M., Werner, O. and Schwarz, W., 2004. Aluminous granulites from the Pulur complex, NE Turkey: a case of partial melting, efficient melt extraction and crystallization. Lithos, 72, 183-207.

Topuz, G., Altherr, R., Siebel, W., Schwarz, W., Zack, T., Hasözbek, A., Barth, M., Satır, M. and Şen, C., 2010. Carboniferous High-potassium I-type Granitoid Magmatism in the Eastern Pontides:The Gümüşhane Pluton (NE Turkey). Lithos, 116, 92-110.

Vd'ačný, M., Madzin, J. and Plašienka, D., 2019. Geochemical characteristics of the Upper Cretaceous to Lower Eocene sedimentary rocks from the Pieniny Klippen Belt (Western Carpathians, Slovakia): implications for tectonic setting, paleoenvironment and paleoclimate. Geosciences Journal, 23 (5), 731-745.

Wang, Y., Long, X., Wilde, A.A., Xu, H., Sun, M. and Xiao, W., 2014. Provenance of Early Paleozoic metasediments in the central Chinese Altai: Implication for tectonic affinity of the Altai-Mongolia terrane in the Central Asian Orogenic Belt. Lithos, 210-211, 57-68.

Wang, Z., Fu, X., Feng, X., Song, C., Wang, D., Chen, W., and Zeng, S., 2017. Geochemical features of the black shales from the Wuyu Basin, southern Tibet: implications for palaeoenvironment and palaeoclimate. Geological Journal, 52, 282297.

White, A.F., Bullen, T.D., Schulz, M.S., Blum, A.E., Huntington, T.G. and Peters, N.E., 2001. Differential rates of feldspar weathering in granitic regoliths. Geochimica et Cosmochimica Acta, 65, 847-869.

Wronkiewicz, D.J. and Condie, K.C., 1987. Geochemistry of Archean shales from the Witwatersrand Supergroup, South Africa: sourc-area weathering and provenance. Geochimica et Cosmochimica Acta, 53, 1537-1549.

Young, G.M., 1999. Some aspects of the geochemistry, provenance and palaeoclimatology of the Torridonian of NW Scotland. J. Geol. Soc. Lond., 156, 10971111.

Zhao, Z., Zhao, J., Wang, H., Liao, J., Liu, C., 2007. Distribution characteristics and applications of trace elements in Junggar Basin (in Chinese with English abstract). Natural Gas Exploration and Development, 30, 30-33.

Zhou, L., Friis, H., Yang, T. and Nielsen, A.N. 2017. Geochemical interpretation of the Precambrian basement and overlying Cambrian sandstone on Bornholm, Denmark: Implications for the weathering history. Lithos, 286-287, 369-387. 